\title{
Web crippling strength of cold-formed stainless steel lipped channel-sections with web openings subjected to Interior-One-Flange loading condition
}

\author{
Amir M. Yousefi ${ }^{1 a}$, James B.P. Lim¹, Asraf Uzzaman²b, Ying Lian ${ }^{3 c}$, \\ 'G Charles Clifton' ${ }^{1 d}$, Ben Young ${ }^{4 e}$ \\ ${ }^{1}$ Department of Civil and Environmental Engineering, The University of Auckland, \\ New Zealand \\ ${ }^{2}$ Department of Mechanical and Aerospace Engineering, The University of Strathclyde, \\ 75 Montrose Street, Glasgow G1 1XJ, UK \\ ${ }^{3}$ SPACE, David Keir Building, Queen's University, Belfast, BT9 5AG, UK \\ ${ }^{4}$ Department of Civil Engineering, The University of Hong Kong, Pokfulam Road, \\ Hong Kong
}

(Received

, Revised

, Accepted

)

\begin{abstract}
In cold-formed stainless steel lipped channel-sections, web openings are becoming increasingly popular. Such openings, however, result in the sections becoming more susceptible to web crippling, especially under concentrated loads applied near the web opening. This paper presents the results of a finite element parametric study into the effect of circular web openings on the web crippling strength of cold-formed stainless steel lipped channel-sections for the interiorone-flange (IOF) loading condition. This involves a bearing load applied to the top flange of a length of member, away from the end supports. The cases of web openings located centred beneath the bearing load (i.e. beneath the bearing plate delivering the load) and offset to the bearing plate, are considered. Three grades of stainless steel are considered: duplex EN1.4462, austenitic EN1.4404 and ferretic EN1.4003. In total, 2218 finite element models were analysed. From the results of the parametric study, strength reduction factors for load bearing capacity are determined, where these reduction factors are applied to the bearing capacity calculated for a web without openings, to take account the influence of the web openings. The strength reduction factors are first compared to equations recently proposed for cold-formed carbon steel lipped channel-sections. It is shown that for the case of the duplex grade, the strength reduction factor equations for coldformed carbon steel are conservative but only by $2 \%$. However, for the cases of the austentic and ferritic grades, the cold-formed carbon steel equations are around $9 \%$ conservative. New strength reduction factor equations are proposed for all three stainless steel grades.
\end{abstract}

Keywords: Cold-formed stainless steel; Lipped channel-section; Web crippling; Finite element analysis; Strength reduction factor.

\footnotetext{
${ }^{a}$ First author, PhD student, Email: ayou561@aucklanduni.ac.nz

*Corresponding author, Senior lecturer, E-mail: james.lim@auckland.ac.nz

${ }^{\mathrm{b}}$ Research Fellow, E-mail: asraf.uzzaman@strath.ac.uk

${ }^{c}$ PhD student, E-mail: ylian01@qub.ac.uk

dAssociate Professor, E-mail: c.clifton@auckland.ac.nz

e Professor, E-mail: young@hku.hk
} 


\begin{tabular}{|ll|}
\hline Nomenclature & \\
$\mathrm{A}$ & Web opening ratio; \\
$a$ & Diameter of circular web opening; \\
$b_{f}$ & Overall flange width of section; \\
$b_{l}$ & Overall lip width of section; \\
$\mathrm{COV}$ & Coefficient of variation; \\
$d$ & Overall web depth of section; \\
$E$ & Young's modulus of elasticity; \\
$h$ & Depth of the flat portion of web; \\
$L$ & Length of the specimen; \\
$N$ & Length of the bearing plate; \\
$P_{\mathrm{ASCE}}$ & Nominal web crippling strength obtained from American Code; \\
$P_{\mathrm{AS} / \mathrm{NZS}}$ & Nominal web crippling strength obtained from Australian/New Zealand Code; \\
$P_{\mathrm{FEA}}$ & Web crippling strength per web predicted from finite element (FEA); \\
$P_{\mathrm{NAS}}$ & Nominal web crippling strength obtained from North American Specification; \\
$P_{m}$ & Mean value of analysed-to-predicted load ratio; \\
$R$ & Reduction factor; \\
$R_{\mathrm{P}}$ & Proposed reduction factor; \\
$r_{\mathrm{i}}$ & Inside corner radius of section; \\
$\theta$ & Angle between web and bearing surface \\
$t$ & Thickness of section; \\
$V_{P}$ & Coefficient of variation of Analysed-to-predicted load ratio; \\
$x$ & Horizontal clear distance of the web openings to the near edge of the bearing plate; \\
$\beta$ & Reliability index;
\end{tabular}

\section{Introduction}

The use of cold-formed stainless steel in structural applications is growing steadily (Dai and Lam 2010, Theofanous and Gardner 2011, Kiymaz and Seckin 2014) and the use of web openings in such sections is becoming increasingly popular (Lawson et. al. 2015) (see Fig. 1). Such openings, however, result in the sections being more susceptible to web crippling, especially under concentrated loads applied to the bearing flange in the vicinity of the openings. No previous research has considered the web crippling strength of coldformed stainless steel lipped channel-sections with circular web openings under either of the one or two-flange loading conditions (see Figs 2 and 3 for the interior-one-flange (IOF) loading condition). For cold-formed stainless steel lipped channel-sections without openings, only Kroyink et al. (1996) has considered the web crippling strength. More recently, Zhou and Young $(2006,2007,2013)$ have considered the web crippling strength of cold-formed stainless steel tubular sections. Research by Lawson et al. (2015), while concerned with circular web openings, focussed on the bending strength of the sections and not on the web crippling strength under concentrated loads. 
For cold-formed carbon steel lipped channel-sections, Lian et al. (2016a,b,c,d,e) recently proposed strength reduction factor equations for sections with circular web openings under oneflange loading; the cases of both flanges fastened and flanges unfastened to the bearing plates were considered. The work of Lian et al. (2016a,b,c,d,e) was a continuation of that of Uzzaman et al. (2012a,b,c, 2013), who considered two-flange loading. The strength reduction factor equations proposed were determined from a parametric study using the finite element program ABAQUS (2014); laboratory test results were used to validate the finite element models. Other recent work reported in the literature includes that of Natario et al. (2015), who proposed a Direct Strength Method (DSM) approach to predict the web crippling strength of cold-formed carbon steel beams under end-two-flange (ETF) loading condition, and Sudarajah et al. (2016) who reported experimental tests and finite element analysis for lipped channel beams (LCBs) under two flange loading conditions.

This paper considers how applicable the equations proposed by Lian et al. $(2016 \mathrm{a}, \mathrm{b}, \mathrm{e})$ for the interior-one-flange (IOF) loading (see Fig. 2), are to three stainless steel grades: duplex grade EN 1.4462; austenitic grade 1.4404 and ferritic grade 1.4003. Typical stress-strain curves for the three grades were taken from Chen and Young (2006) and Arrayago et. al. (2015). Comparative hotrolled steel stress strain curves can be found in Yousefi et al. (2014) and Rezvani et al. (2015).

\section{Experimental investigation and finite element modelling}

For cold-formed carbon steel, Lian et al. (2016a,b,e) recently conducted 43 interior-one-flange (IOF) laboratory tests on lipped channel-sections with circular web openings subjected to web crippling (see Fig. 3). Fig. 4 shows the definition of the symbols used to describe the dimensions of the cold-formed carbon steel lipped channel-sections considered in the test programme. The laboratory tests were used to validate a non-linear geometry elasto-plastic finite element model in ABAQUS (2014), which was then used for a parametric study, from which design recommendations were proposed in the form of strength reduction factor equations, relating the loss of strength due to the web openings to the strength of the web without openings. The size of the circular web openings was varied in order to investigate the effect of the web opening size on the web crippling strength. Full details of both the laboratory tests and finite element models (see Fig. 5) can be found in Lian et al. (2016a,b,e). However, brief details of the finite element models are summarised below.

In the finite element model, one-quarter of the test set-up of Lian et al. (2016a,b,e) was modelled, as shown in Fig 5. In ABAQUS, the S4R shell element was used. The S4R is a four-node double curved thin or thick shell element with reduced integration and finite membrane strains. The S4R has six degrees of freedom per node and provides accurate solutions to most applications. The bearing plates and load transfer block were modelled using analytical rigid plates and using C3D8R element, which is suitable for three-dimensional modelling of structures with plasticity, stress stiffening, large deflection, and large strain capabilities. The solid element is defined by eight nodes having three translational degrees of freedom at each node.

Contact between the bearing plate, the load transfer block and the cold-formed steel section was modelled in ABAQUS using the contact pairs option. The two contact surfaces were not allowed to penetrate each other. No friction was modelled between the surfaces. The lipped channel-sections of the model were based on the centreline dimensions of the cross-sections. Finite element mesh sizes were $5 \mathrm{~mm} \times 5 \mathrm{~mm}$ for the cold-formed steel channel sections and $8 \mathrm{~mm} \times 8 \mathrm{~mm}$ for the bearing plates and load transfer blocks. The vertical load applied to the channel section through the 
bearing plate in the laboratory tests was modelled using displacement control. In the finite element model, a displacement in the vertical y direction was applied to the reference point of the analytical rigid plate that modelled the bearing plate. At the line of symmetry of the channel section, all nodes were restrained in the $\mathrm{z}$ direction and rotation about $\mathrm{x}$ and $\mathrm{y}$ axes. The nodes on symmetry surface of load transfer block were prevented from translational axes in the $\mathrm{x}$ direction and rotation about the $y$ and $\mathrm{z}$ axes. The channel section specimens were tested in pairs, which were bolted to load transfer blocks at each end of the specimens through the web by a vertical row of M16 high tensile bolts. In the shell element idealisation, cartesian connectors with an in-plane stiffness were used to simulate bolt-hole elongation instead of physically modelling bolts and holes. "CONN3D2" connector elements were used to model the in-plane translational stiffness i.e. y- and z-directions. The stiffness of the connectors element was $10 \mathrm{kN} / \mathrm{mm}$, which Lim and Nethercot (2001) suggest would be suitable. In the $\mathrm{x}$ direction, the nodes were prevented from translating.

The models have been coded such the nominal dimension of the model and the length of the bearing plate as well as the ratio of the diameter of the circular web openings to the depth of the flat portion of the webs $(a / h)$ can be determined from the coding system. As an example, the label "142-N100-A0.2-FR" means the following. The first notation is the nominal depth of the models in millimeters. The notation "N1OO" indicates the length of bearing in millimeters (i.e. $100 \mathrm{~mm}$ ). The notation " $A 0.2$ " indicates the ratio of the diameter of the openings to the depth of the flat portion of the webs $(a / h)$ and are one of 0.2, 0.4, 0.6 and 0.8 (i.e. $A 0.2$ means $a / h=0.2 ; A 0.4$ means $a / h=$ 0.4 etc). Plain lipped channel-sections (i.e. without circular web openings) are denoted by " $A O$ ". The flange unfastened and fastened cases are identified as " $F R$ " and " $F X$ ", respectively.

Fig. 6 compares the experimental and numerical load-displacement curves for a cold-formed carbon steel lipped channel-section, $142 \times 60 \times 13-\mathrm{t} 1.3-\mathrm{N} 100$-FR, covering the cases both with and without the circular web openings. As can be seen, there is good agreement between the failure loads of the tested specimens and the finite element results.

For cold-formed stainless steel lipped channel-sections, the numerical failure loads with and without circular web openings were then determined for the three stainless steel grades: duplex grade EN 1.4462; austenitic grade 1.4404 and ferritic grade 1.4003 (see Table 1). These results were compared with the failure loads calculated in accordance with ASCE (2002), NAS (2007) and AS/NZS 4600 (2005) (see Table 2). The failure loads predicted from the finite element model are similar to the codified failure loads of the sections.

\section{Parametric study for stainless steel grades}

In this study, in order to investigate the effect of circular web openings on the web crippling strength of cold-formed stainless steel lipped channel-sections, a total of 2218 finite element models of lipped channel-sections with various dimensions and thicknesses were considered for the three stainless steel grades: duplex EN1.4462, austenitic EN1.4404 and ferretic EN1.4003. Table 3 shows the web crippling strengths determined from finite element analyses for the duplex grade EN 1.4462. Similar web crippling strengths were also determined for the austenitic grade 1.4404 and ferritic grade 1.4003 . The web crippling strengths for sections with circular web openings were divided by that for sections without web openings and considered as the strength reduction factor (R).

Fig. 7 shows the ratio of the circular web opening depth to the flat portion of the web $(a / h)$ versus the strength reduction factor, for the three stainless steel grades. As can be seen, the reduction in strength increases as the parameter $a / h$ increases for all three stainless steels, in particular for the 
ferritic grade. The reduction in strength of the ferritic grade $6 \mathrm{~mm}$ thick section is smallest and the reduction in strength increases as the section becomes thinner. It can be seen that when the $a / h$ ratio increases from 0.2 to 0.8 , the reduction in strength for the ferritic grade increases by $36 \%$. From Fig. 8 it can be seen that the reduction in strength is sensitive to the horizontal distance of the web openings to the bearing plate. As the ratio of $x / h$ decreases from 0.6 to 0.2 , the strength reduction factor decreases by $7 \%$. Also, it can again be seen that the reduction in strength is less for the austenitic grade compared to that of the other two stainless steel grades.

\section{Reduction factor comparison with Lian et al. (2016a,b,e)}

For ease of reference, the reduction factor equations proposed by Lian et al. $(2016 \mathrm{a}, \mathrm{b}, \mathrm{e})$ are summarised below:

For centered web opening:

Free case $\quad R_{P}=0.98-0.26\left(\frac{a}{h}\right)+0.06\left(\frac{N}{h}\right)$

Fixed case $\quad R_{P}=0.95-0.06\left(\frac{a}{h}\right)+0.01\left(\frac{N}{h}\right)$

For offset web opening:

Free case $\quad R_{P}=0.99-0.26\left(\frac{a}{h}\right)+0.11\left(\frac{x}{h}\right)$

Fixed case: $R_{P}=0.99-0.14\left(\frac{a}{h}\right)+0.07\left(\frac{x}{h}\right)$

where the limits for the reduction factor in Eqs. (1)-(4) are $h / t \leq 157.8, N / t \leq 120.97$, $N / h \leq 1.15, a / h \leq 0.8$, and $\theta=90^{\circ}$.

In order to evaluate the applicability of the proposed equations to cold-formed stainless steel grades, an extensive statistical analysis was performed on all four proposed equations. Table 5 compares the reduction factors determined from the finite element models to Eqs. (1)-(4) for cases of centred and offset web opening where the flange is unfastened to the bearing plate.

As can be seen from Table 5, the four equations proposed by Lian et al. (2016a,b,e) for carbon steel are conservative for the three stainless steel grades, especially for sections with centred web openings. Examining the strength reduction factor ratios obtained from the finite element analyses, with the exception of the offset web opening fixed case which has a mean reduction factor ratio of 0.99 and coefficient of variation (COV) of 0.05 , the other reduction factors from Lian et al. (2016a,b,e) are conservative for the stainless steel grades, especially for the ferritic and austenitic stainless steel grades. For example, for the centred web opening case for austenitic grade, the mean value of the web crippling reduction factor ratio is 1.09 and 1.07 for the cases of flange unfastened and fastened to the bearing plate, respectively; the corresponding values of COV are 0.04 and 0.01 , respectively. In the next section, new equations are proposed for each of the three stainless steel grades.

\section{Proposed strength reduction factors}

Table 3 shows the dimensions considered and web crippling strengths of the duplex grade stainless steel sections predicted from the finite element analysis. (Tables for the austenitic and 
ferritic stainless steel grades can be found in Appendices A and B). Using bivariate linear regression analysis, two unified strength reduction factor equations $\left(R_{\mathrm{p}}\right)$ for three stainless steel grades with web openings are proposed. The equations are as follows:

$$
\begin{array}{ll}
\text { Centred web opening } & R_{p}=\alpha-\gamma\left(\frac{a}{h}\right)-\lambda\left(\frac{N}{h}\right) \leq 1 \\
\text { Offset web opening } & R_{p}=\rho+\mu\left(\frac{a}{h}\right)+\zeta\left(\frac{x}{h}\right) \leq 1
\end{array}
$$

The limits for the reduction factor Eqs. (5)-(6) remain $h / t \leq 157.8, N / t \leq 120.97$, $N / h \leq 1.15, a / h \leq 0.8$, and $\theta=90^{\circ}$. The coefficients $\alpha, \gamma, \lambda, \rho, \mu$ and $\zeta$ of the equations are calibrated with the stainless steel analysis results, and the coefficients are presented in Table 4.

\section{Comparison of numerical results with proposed reduction factors}

For the three stainless steels grades, the values of the strength reduction factor $(R)$ obtained from the numerical results are compared with the values of the proposed strength reduction factor $\left(R_{p}\right)$ calculated using Eqs. (5)-(6). The results for C142 are shown in Figs 9 and 10. In order to evaluate the accuracy of proposed equations, extensive statistical reliability analyses are performed. The results are summarized in Table 6 . It should be noted, in calculating the reliability index, the resistance factor of $\phi=0.85$ was used, corresponding to the reliability index $\beta$ from the NAS specification. According to the NAS specification, design rules are reliable if the reliability index are more than 2.5. As can be seen in Table 6, the proposed reduction factors are a good match with the numerical results for the both cases of flanges unfastened and flanges fastened to the bearing plates and particularly for the duplex stainless steel grade.

For example, for the centred circular web opening, the mean value of the web crippling reduction factor ratios are 1.00 and 1.01 for the cases of flange unfastened and flange fastened to the bearing plate, respectively. The corresponding values of COV are 0.03 and 0.03 , respectively. Similarly, the reliability index values $(\beta)$ are 2.82 and 2.86, respectively. For the offset circular web opening, the mean value of the web crippling reduction factor ratios are 1.04 and 1.04 for the cases of flange unfastened and flange fastened to the bearing plate, respectively. The corresponding values of COV are 0.04 and 0.05 , respectively. Similarly, the reliability index values $(\beta)$ are 2.97 and 2.94, respectively. Therefore, the proposed strength reduction factor equations are able to reliably predict the influence of the circular web openings on the web crippling strengths of coldformed stainless steel lipped channel-sections under the interior-one-flange (IOF) loading condition.

\section{Conclusions}

In this study, the effect of circular web openings on the web crippling strength of cold-formed stainless steel lipped channel-sections was investigated. For this purpose, a parametric study of 2218 lipped channel-sections with various dimensions and thicknesses were considered for the three stainless steel grades: duplex EN1.4462, austenitic EN1.4404 and ferretic EN1.4003. Cases with and without circular web openings subjected to the interior-one-flange (IOF) loading condition were considered with web openings located centred beneath the bearing plate or with a horizontal clear distance to the near edge of the bearing plate. 
- In order to take into account the influence of the circular web openings, strength reduction factor equations were determined. Then, the strength reduction factor equations were compared to recent equations proposed for cold-formed carbon steel. It was observed that the cold-formed carbon steel strength reduction factors are conservative to the duplex grade by around $2 \%$ conservative to the austenitic and ferritic grades by around $9 \%$.

- New web crippling strength reduction factor equations were proposed for the both cases of flanges unfastened and flanges fastened to the bearing plates.

- In order to evaluate the reliability of proposed reduction factors, reliability analysis was performed. It was demonstrated that the proposed strength reduction factors are generally conservative and agree well with the analysis results. The proposed new strength reduction factors have the capability to produce safe yet not too conservative design values when calibrated with the resistance factor of $0.85(\varphi=0.85)$ according to the NAS specification.

\section{References}

ABAQUS. (2014). Analysis User's Manual-Version 6.14-2 ABAQUS Inc., USA.

Arrayago, I., E. Real, L. Gardner (2015), "Description of stress-strain curves for stainless steel alloys", Materials \& Design, 87, 540-552.

AS/NZS 4600 (2005), Cold-formed steel structures: AS/NZS 4600:2005, Standards Australia, Sydney, Australia.

ASCE 8-02 (2002), Specification for the Design of Cold-Formed Stainless Steel Structural Members: SEI/ASCE 8-02, Reston, VA.

Chen, J., and Ben Young (2006), "Stress-strain curves for stainless steel at elevated temperatures", Engineering Structures, 28(2), 229-239.

Dai, X., and D. Lam (2010), "Axial compressive behaviour of stub concrete-filled columns with elliptical stainless steel hollow sections", Steel and Composite Structures 10(6), 517-539.

Kiymaz, G., and Edip Seckin (2014), "Behavior and design of stainless steel tubular member welded end connections", Steel and Composite Structures 17(3), 253-269.

Korvink, S.A., G.J. van den Berg, P. van der Merwe (1995), "Web crippling of stainless steel cold-formed beams", Journal of Constructional Steel Research, 34 (2-3), 225-248.

Lawson, R. M., Antonio Basta, Assraf Uzzaman (2015), "Design of stainless steel sections with circular openings in shear", Journal of Constructional Steel Research, 112, 228-241.

Lian, Y., Uzzaman A., Lim J.B.P., Abdelal G., Nash D., Young B. (2016a), "Web Crippling Behaviour of Cold-Formed Steel Channel Sections with Web Holes Subjected to Interior-One-Flange Loading Condition - Part I: Experimental and Numerical Investigations", Submitted to Thin-Walled Structures.

Lian, Y., Uzzaman A., Lim J.B.P., Abdelal G., Nash D., Young B. (2016b), "Web Crippling Behaviour of Cold-Formed Steel Channel Sections with Web Holes Subjected to Interior-One-Flange Loading Condition - Part II: Parametric Study and Proposed Design Equations", Submitted to Thin-Walled Structures.

Lian, Y., Uzzaman A., Lim J.B.P., Abdelal G., Nash D., Young B. (2016c), "Effect of web holes on web crippling strength of cold-formed steel channel sections under end-one-flange loading condition - Part I: Tests and finite element analysis", Submitted to Thin-Walled Structures.

Lian, Y., Uzzaman A., Lim J.B.P., Abdelal G., Nash D., Young B. (2016d), "Effect of web holes on web crippling strength of cold-formed steel channel sections under end-one-flange loading condition - Part II: Parametric study and proposed design equations", Submitted to Thin-Walled Structures. 
Lian, Y. (2016e), "Web crippling behaviour of cold-formed steel sections with circular web openings under one-flange loading", PhD Thesis, School of Planning, Architecture and Civil Engineering, The Queen's University of Belfast, Northern Ireland.

Lim J.B.P, Nethercot D.A. (2001), "Ultimate strength of bolted moment-connections between cold-formed steel members", Thin Walled Structures, 41 (2001) 1019-1039.

NAS (2007), North American Specification for the Design of Cold-Formed Steel Structural Members: American Iron and Steel Institute, AISI S100-2007, AISI Standard.

Natário, P., N. Silvestre, D. Camotim (2016), "Direct strength prediction of web crippling failure of beams under ETF loading", Thin-Walled Structures, 98(B), 360-374.

Rezvani, F. H., Yousefi, A. M., \& Ronagh, H. R. (2015), "Effect of span length on progressive collapse behaviour of steel moment resisting frames". Structures, 3, 81-89.

Sundararajah, L., Mahen Mahendran, Poologanathan Keerthan (2016). "Experimental Studies of Lipped Channel Beams Subject to Web Crippling under Two-Flange Load Cases." Journal of Structural Engineering.

Theofanous, M., and L. Gardner (2012), "Effect of element interaction and material nonlinearity on the ultimate capacity of stainless steel cross-sections", Steel and Composite Structures, 12 (1), 73-92.

Uzzaman, A., James B.P. Lim, David Nash, Jim Rhodes, Ben Young (2012a), "Web crippling behaviour of cold-formed steel channel sections with offset web holes subjected to interior-two-flange loading", Thin-Walled Structures, 50, 76-86.

Uzzaman, A., James B.P. Lim, David Nash, Jim Rhodes, Ben Young (2012b), "Cold-formed steel sections with web openings subjected to web crippling under two-flange loading conditions-part I: Tests and finite element analysis", Thin-Walled Structures, 56, 38-48.

Uzzaman, A., James B.P. Lim, David Nash, Jim Rhodes, Ben Young (2012c), "Cold-formed steel sections with web openings subjected to web crippling under two-flange loading conditions-part II: Parametric study and proposed design equations", Thin-Walled Structures, 56, 79-87.

Uzzaman, A., James B.P. Lim, David Nash, Jim Rhodes, Ben Young (2013), "Effect of offset web holes on web crippling strength of cold-formed steel channel sections under end-two-flange loading condition", Thin-Walled Structures, 65, 34-48.

Yousefi, A. M., Mojtaba Hosseini and Nader Fanaie (2014), Vulnerability Assessment of Progressive Collapse of Steel Moment Resistant Frames. Trends in Applied Sciences Research, 9: 450-460.

Zhou, F., and Ben Young (2006), "Yield line mechanism analysis on web crippling of cold-formed stainless steel tubular sections under two-flange loading", Engineering Structures, 28(6), 880-892.

Zhou, F., and Ben Young (2007), "Cold-formed high-strength stainless steel tubular sections subjected to web crippling", Journal of structural engineering, 133(3), 368-377.

Zhou, F., and Ben Young (2013), "Web crippling behaviour of cold-formed duplex stainless steel tubular sections at elevated temperatures", Engineering Structures, 57, 51-62. 


\section{List of tables}

Table 1 Dimensions and web crippling strengths predicted from finite element analysis of coldformed stainless steel lipped channel sections

(a) For the case of flange unfastened to the bearing plate

(b) For the case of flange fastened to the bearing plate

Table 2 Comparison of numerical results with design strength for the case of flange fastened to the bearing plate without circular web opening

Table 3 Web crippling strengths of duplex stainless steel sections predicted from finite element analysis
(a) $a / h$ for centred circular web opening case
(b) $a / h$ for offset circular web opening case
(c) $x / h$ for offset circular web opening case

Table 4 Coefficients of the proposed strength reduction factor equations

Table 5 Comparison of web crippling strength reduction factor for cold-formed stainless steel lipped channel sections with reduction factor equations proposed by Lian et al. (2016a,b,e)

(a) Flange unfastened to the bearing plate

(b) Flange fastened to the bearing plate

Table 6 Statistical analysis of strength reduction factor

(a) Duplex stainless steel grade

(b) Austenitic stainless steel grade

(c) Ferritic stainless steel grade 
Table 1 Dimensions and web crippling strengths predicted from finite element analysis of cold-formed stainless steel lipped channel-sections (a) For the case of flange unfastened to the bearing plate

\begin{tabular}{|c|c|c|c|c|c|c|c|c|c|c|c|c|c|c|c|}
\hline \multirow[t]{2}{*}{ Specimen } & \multirow{2}{*}{$\begin{array}{c}\text { Web } \\
d \\
(\mathrm{~mm})\end{array}$} & \multirow{2}{*}{$\begin{array}{c}\text { Flange } \\
\qquad \begin{array}{c}b_{f} \\
(\mathrm{~mm})\end{array}\end{array}$} & \multirow{2}{*}{$\begin{array}{l}\text { Lip } \\
\quad b_{l} \\
(\mathrm{~mm})\end{array}$} & \multirow{2}{*}{$\begin{array}{c}\text { Thickness } \\
t \\
(\mathrm{~mm})\end{array}$} & \multirow{2}{*}{$\begin{array}{c}\text { Length } \\
\qquad \\
L \\
(\mathrm{~mm})\end{array}$} & \multirow{2}{*}{$\begin{array}{c}\text { Web } \\
\text { opening } \\
a \\
(\mathrm{~mm})\end{array}$} & \multicolumn{3}{|c|}{ Duplex } & \multicolumn{3}{|c|}{ Ferritic } & \multicolumn{3}{|c|}{ Austenitic } \\
\hline & & & & & & & $\begin{array}{c}P(A 0) \\
(k N)\end{array}$ & $\begin{array}{l}\text { P(Opening) } \\
(k N)\end{array}$ & $\begin{array}{l}P(\text { Opening }) \\
(k N)\end{array}$ & $\begin{array}{l}P(A 0) \\
(k N)\end{array}$ & $\begin{array}{l}\text { P(Opening }) \\
(k N)\end{array}$ & $\begin{array}{l}\text { P(Opening }) \\
(k N)\end{array}$ & $\begin{array}{l}P(A 0) \\
(k N)\end{array}$ & $\begin{array}{l}\text { P(Opening }) \\
(k N)\end{array}$ & $\begin{array}{l}\text { P(Opening }) \\
(\mathrm{kN})\end{array}$ \\
\hline & & & & & & & & Offset & Centred & & Offset & Centred & & Offset & Centred \\
\hline 142-N100-MA0.6-FR & 141.82 & 60.63 & 13.66 & 1.27 & 720.00 & 139.27 & 11.57 & 10.95 & 10.41 & 9.64 & 9.40 & 8.98 & 8.52 & 8.14 & 7.81 \\
\hline 142-N120-MA0.6-FR & 142.24 & 60.37 & 13.90 & 1.27 & 740.00 & 139.70 & 12.28 & 11.41 & 10.77 & 10.30 & 10.02 & 9.38 & 8.90 & 8.66 & 8.21 \\
\hline 202-N100-MA0.4-FR & 202.04 & 64.79 & 14.78 & 1.38 & 899.20 & 199.28 & 12.56 & 11.44 & 11.96 & 10.95 & 10.44 & 10.92 & 9.60 & 7.95 & 9.57 \\
\hline 202-N100-MA0.6-FR & 202.04 & 64.79 & 14.78 & 1.38 & 899.20 & 199.28 & 12.55 & 10.37 & 12.47 & 10.95 & 9.83 & 10.93 & 9.60 & 7.76 & 9.58 \\
\hline 202-N120-MA0.4-FR & 202.00 & 65.00 & 14.73 & 1.38 & 920.00 & 199.24 & 12.81 & 11.65 & 12.09 & 11.59 & 10.88 & 11.25 & 10.15 & 9.71 & 10.10 \\
\hline 202-N120-MA0.6-FR & 202.00 & 65.00 & 14.73 & 1.38 & 920.00 & 199.24 & 12.81 & 10.51 & 10.80 & 11.59 & 10.03 & 10.05 & 10.15 & 9.48 & 9.31 \\
\hline 202-N150-MA0.4-FR & 202.01 & 65.04 & 14.98 & 1.38 & 950.00 & 199.24 & 13.15 & 11.93 & 12.26 & 12.25 & 11.27 & 11.52 & 10.90 & 10.49 & 10.65 \\
\hline 302-N120-MA0.6-FR & 303.07 & 87.95 & 18.26 & 1.90 & 1221.00 & 299.26 & 26.01 & 21.95 & 25.30 & 22.47 & 20.18 & 22.16 & 19.41 & 17.66 & 19.30 \\
\hline 302-N150-MA0.6-FR & 303.03 & 88.54 & 18.97 & 1.90 & 1249.00 & 299.23 & 27.71 & 22.58 & 27.51 & 24.65 & 21.50 & 24.55 & 21.50 & 19.26 & 21.45 \\
\hline
\end{tabular}


(b) For the case of flange fastened to the bearing plate

\begin{tabular}{|c|c|c|c|c|c|c|c|c|c|c|c|c|c|c|c|}
\hline \multirow[t]{2}{*}{ Specimen } & \multirow{2}{*}{$\begin{array}{c}\text { Web } \\
\begin{array}{c}d \\
(\mathrm{~mm})\end{array}\end{array}$} & \multirow{2}{*}{$\begin{array}{c}\text { Flange } \\
\qquad \begin{array}{c}b_{f} \\
(\mathrm{~mm})\end{array}\end{array}$} & \multirow{2}{*}{$\begin{array}{l}\text { Lip } \\
\quad b_{l} \\
(\mathrm{~mm})\end{array}$} & \multirow{2}{*}{$\begin{array}{c}\text { Thickness } \\
t \\
(\mathrm{~mm})\end{array}$} & \multirow{2}{*}{$\begin{array}{c}\text { Length } \\
\\
L \\
(\mathrm{~mm})\end{array}$} & \multirow{2}{*}{$\begin{array}{c}\text { Web } \\
\text { opening } \\
a \\
(\mathrm{~mm})\end{array}$} & \multicolumn{3}{|c|}{ Duplex } & \multicolumn{3}{|c|}{ Ferritic } & \multicolumn{3}{|c|}{ Austenitic } \\
\hline & & & & & & & $\begin{array}{c}P(A 0) \\
(k N)\end{array}$ & $\begin{array}{l}\text { P(Opening) } \\
(k N)\end{array}$ & $\begin{array}{l}P(\text { Opening }) \\
(k N)\end{array}$ & $\begin{array}{l}P(A 0) \\
(k N)\end{array}$ & $\begin{array}{l}P(\text { Opening }) \\
(k N)\end{array}$ & $\begin{array}{l}\text { P(Opening) } \\
(k N)\end{array}$ & $\begin{array}{l}P(A 0) \\
(k N)\end{array}$ & $\begin{array}{l}\text { P(Opening }) \\
(k N)\end{array}$ & $\begin{array}{l}P(\text { Opening }) \\
(k N)\end{array}$ \\
\hline & & & & & & & & Offset & Centred & & Offset & Centred & & Offset & Centred \\
\hline 142-N100-MA0.6-FX & 142.49 & 60.33 & 13.79 & 1.29 & 720.00 & 139.27 & 12.76 & 12.53 & 11.66 & 10.13 & 10.15 & 9.67 & 9.40 & 9.24 & 8.64 \\
\hline 142-N120-MA0.6-FX & 142.38 & 60.21 & 13.68 & 1.29 & 740.00 & 139.70 & 13.49 & 13.38 & 12.07 & 11.01 & 10.96 & 10.17 & 9.86 & 9.76 & 9.04 \\
\hline 142-N150-MA0.4-FX & 142.18 & 60.12 & 13.19 & 1.28 & 770.00 & 139.84 & 14.37 & 14.34 & 13.80 & 11.91 & 11.91 & 11.64 & 10.70 & 10.47 & 10.46 \\
\hline 202-N100-MA0.4-FX & 201.99 & 64.87 & 14.76 & 1.37 & 900.00 & 199.28 & 13.51 & 14.17 & 13.49 & 10.99 & 11.57 & 10.56 & 10.08 & 10.51 & 10.01 \\
\hline 202-N100-MA0.6-FX & 201.99 & 64.87 & 14.76 & 1.37 & 900.00 & 199.28 & 13.51 & 14.03 & 12.60 & 10.99 & 11.50 & 10.47 & 10.08 & 10.38 & 9.88 \\
\hline 202-N120-MA0.4-FX & 202.05 & 64.99 & 14.82 & 1.41 & 920.00 & 199.24 & 15.18 & 14.24 & 15.14 & 12.51 & 11.82 & 12.24 & 11.20 & 10.51 & 10.97 \\
\hline 202-N120-MA0.6-FX & 202.05 & 64.99 & 14.82 & 1.41 & 920.00 & 199.24 & 15.18 & 14.06 & 13.60 & 12.51 & 11.70 & 11.90 & 11.20 & 10.39 & 10.71 \\
\hline 202-N150-MA0.4-FX & 202.00 & 64.93 & 15.00 & 1.41 & 950.00 & 199.24 & 16.45 & 16.30 & 16.05 & 13.73 & 13.69 & 13.14 & 12.23 & 12.09 & 11.58 \\
\hline 302-N100-MA0.6-FX & 303.20 & 88.24 & 18.66 & 1.96 & 1199.00 & 299.37 & 26.27 & 25.72 & 23.37 & 20.91 & 20.78 & 19.91 & 19.25 & 19.05 & 17.93 \\
\hline 302-N120-MA0.6-FX & 303.50 & 88.53 & 18.36 & 1.93 & 1219.00 & 299.26 & 27.17 & 26.57 & 23.49 & 21.96 & 21.84 & 20.62 & 19.75 & 19.66 & 18.59 \\
\hline 302-N150-MA0.6-FX & 303.85 & 88.71 & 18.41 & 1.90 & 1248.33 & 299.23 & 28.56 & 27.19 & 24.25 & 23.59 & 23.41 & 21.28 & 21.06 & 20.46 & 19.31 \\
\hline
\end{tabular}




\begin{tabular}{|c|c|c|c|c|c|c|c|c|c|c|c|}
\hline \multirow[t]{2}{*}{ Specimen } & \multirow{2}{*}{$\begin{array}{l}\text { Web } \\
\text { slenderness } \\
h / t\end{array}$} & \multirow{2}{*}{$\begin{array}{l}\text { Bearing } \\
\text { length to } \\
\text { thickness } \\
\text { ratio } \\
N / t\end{array}$} & \multirow{2}{*}{$\begin{array}{l}\text { Bearing } \\
\text { length to } \\
\text { web height } \\
\text { ratio } \\
N / h\end{array}$} & \multirow{2}{*}{$\begin{array}{l}\text { Inside bend } \\
\text { radius to } \\
\text { thickness } \\
\text { ratio } \\
r_{i} / t\end{array}$} & \multirow{2}{*}{$\begin{array}{l}\text { Failure } \\
\text { load per } \\
\text { web }\end{array}$} & \multicolumn{4}{|c|}{$\begin{array}{l}\text { Web crippling strength per } \\
\text { web predicted from current } \\
\text { design codes }\end{array}$} & \multicolumn{2}{|c|}{ Comparison } \\
\hline & & & & & & $\begin{array}{l}\mathrm{P}_{\mathrm{NAS}} \\
(k N)\end{array}$ & $\begin{array}{l}\mathrm{P}_{\mathrm{ASCE}} \\
(\mathrm{kN})\end{array}$ & $\mathrm{P}_{\mathrm{AS} / \mathrm{NZS}}$ & $\mathrm{P} / \mathrm{P}_{\mathrm{NAS}}$ & $\mathrm{P} / \mathrm{P}_{\mathrm{ASCE}}$ & $\mathrm{P} / \mathrm{P}_{\mathrm{AS} / \mathrm{NZS}}$ \\
\hline 142x60x13-t1.3-N100-A0-FX & 110.19 & 78.74 & 0.71 & 3.78 & 10.13 & 9.23 & 10.13 & 9.22 & 1.10 & 1.00 & 1.10 \\
\hline 142x60x13-t1.3-N120-A0-FX & 110.11 & 94.49 & 0.86 & 3.78 & 11.01 & 9.72 & 11.21 & 9.72 & 1.13 & 0.98 & 1.13 \\
\hline 142x60x13-t1.3-N150-A0-FX & 109.08 & 117.19 & 1.07 & 3.75 & 11.91 & 10.16 & 12.56 & 10.15 & 1.17 & 0.95 & 1.17 \\
\hline 202x65x15-t1.4-N100-A0-FX & 144.37 & 72.46 & 0.50 & 3.62 & 10.99 & 10.08 & 10.37 & 10.08 & 1.09 & 1.06 & 1.09 \\
\hline 202x65x15-t1.4-N120-A0-FX & 144.41 & 86.96 & 0.60 & 3.62 & 12.51 & 11.35 & 12.21 & 11.35 & 1.10 & 1.02 & 1.10 \\
\hline 202x65x15-t1.4-N150-A0-FX & 144.38 & 108.70 & 0.75 & 3.62 & 13.73 & 12.11 & 13.92 & 12.10 & 1.13 & 0.99 & 1.13 \\
\hline $302 \times 90 \times 18-\mathrm{t} 2.0-\mathrm{N} 100-\mathrm{A} 0-\mathrm{FX}$ & 157.58 & 52.63 & 0.33 & 2.63 & 20.91 & 20.67 & 19.46 & 20.67 & 1.01 & 1.07 & 1.01 \\
\hline $302 \times 90 \times 18-t 2.0-\mathrm{N} 120-\mathrm{A} 0-\mathrm{FX}$ & 157.74 & 63.16 & 0.40 & 2.63 & 21.96 & 20.91 & 19.86 & 20.91 & 1.05 & 1.11 & 1.05 \\
\hline $302 \times 90 \times 18-\mathrm{t} 2.0-\mathrm{N} 150-\mathrm{A} 0-\mathrm{FX}$ & 155.43 & 77.72 & 0.50 & 2.59 & 23.59 & 21.34 & 21.41 & 21.32 & 1.11 & 1.10 & 1.11 \\
\hline Mean, Pm & & & & & & & & & 1.10 & 1.03 & 1.09 \\
\hline Coefficient of variation & & & & & & & & & 0.09 & 0.05 & 0.04 \\
\hline
\end{tabular}


Table 3 Web crippling strengths of duplex stainless steel sections predicted from finite element analysis (a) $a / h$ for centred circular web opening case

\begin{tabular}{|c|c|c|c|c|c|c|c|c|c|c|c|}
\hline \multirow[t]{3}{*}{ Specimen } & \multirow{3}{*}{$\begin{array}{c}\text { Thickness } \\
t \\
(\mathrm{~mm})\end{array}$} & \multicolumn{5}{|c|}{ Unfastened FEA load per web, $\mathrm{P}_{\mathrm{FEA}}$} & \multicolumn{5}{|c|}{ Fastened FEA load per web, $\mathrm{P}_{\mathrm{FEA}}$} \\
\hline & & $A(0)$ & $A(0.2)$ & $A(0.4)$ & $A(0.6)$ & $A(0.8)$ & $A(0)$ & $A(0.2)$ & $A(0.4)$ & $A(0.6)$ & $A(0.8)$ \\
\hline & & $(k N)$ & $(k N)$ & $(k N)$ & $(k N)$ & $(k N)$ & $(k N)$ & $(k N)$ & $(k N)$ & $(k N)$ & $(k N)$ \\
\hline 142-N100-FR & 1.27 & 11.57 & 11.50 & 11.45 & 10.41 & 9.10 & 12.76 & 12.72 & 12.72 & 11.66 & 9.98 \\
\hline 142-N100-FR & 4.00 & 93.12 & 91.83 & 86.44 & 80.68 & 71.84 & 112.57 & 111.80 & 105.57 & 95.87 & 86.45 \\
\hline $142-\mathrm{N} 100-\mathrm{FR}$ & 6.00 & 174.70 & 171.91 & 162.61 & 147.12 & 122.67 & 201.87 & 199.27 & 191.73 & 178.14 & 171.28 \\
\hline 142-N120-FR & 1.27 & 12.28 & 12.19 & 11.83 & 10.77 & 9.53 & 13.49 & 13.48 & 13.37 & 12.07 & 10.59 \\
\hline 142-N120-FR & 4.00 & 97.41 & 95.77 & 90.95 & 85.47 & 74.45 & 120.57 & 119.45 & 112.55 & 103.25 & 91.75 \\
\hline 142-N120-FR & 6.00 & 173.11 & 170.45 & 161.34 & 143.15 & 119.29 & 201.42 & 199.08 & 191.99 & 179.79 & 160.09 \\
\hline 142-N150-FR & 1.28 & 12.94 & 12.94 & 12.26 & 11.18 & 10.02 & 14.37 & 14.35 & 13.80 & 12.52 & 11.12 \\
\hline 142-N150-FR & 4.00 & 97.86 & 96.56 & 92.51 & 81.59 & 68.18 & 128.76 & 127.50 & 121.49 & 112.61 & 100.54 \\
\hline 142-N150-FR & 6.00 & 162.37 & 158.99 & 148.73 & 131.58 & 110.03 & 197.41 & 195.84 & 190.05 & 179.26 & 162.44 \\
\hline 202-N100-FR & 1.39 & 12.56 & 12.47 & 11.96 & 10.63 & - & 13.51 & 13.50 & 13.49 & 12.60 & - \\
\hline 202-N100-FR & 4.00 & 93.07 & 92.40 & 88.79 & 80.58 & - & 108.50 & 107.91 & 104.75 & 93.06 & - \\
\hline 202-N100-FR & 6.00 & 188.63 & 184.77 & 173.76 & 158.15 & - & 227.47 & 226.40 & 217.24 & 195.81 & - \\
\hline 202-N120-FR & 1.39 & 12.81 & 12.71 & 12.09 & 10.81 & - & 15.18 & 15.17 & 15.14 & 13.60 & - \\
\hline 202-N120-FR & 4.00 & 97.11 & 96.36 & 91.70 & 83.85 & - & 116.44 & 115.64 & 109.98 & 98.16 & - \\
\hline 202-N120-FR & 6.00 & 191.80 & 188.23 & 177.94 & 160.81 & - & 230.69 & 229.94 & 221.98 & 203.79 & - \\
\hline 202-N150-FR & 1.39 & 13.15 & 13.02 & 12.27 & 11.14 & - & 16.45 & 16.45 & 16.05 & 14.17 & - \\
\hline 202-N150-FR & 4.00 & 102.42 & 101.16 & 95.83 & 89.27 & - & 128.20 & 126.06 & 117.79 & 106.42 & - \\
\hline 202-N150-FR & 6.00 & 188.24 & 186.47 & 179.66 & 159.06 & - & 238.28 & 229.36 & 222.60 & 207.03 & - \\
\hline 302-N100-FR & 1.98 & 24.64 & 24.63 & 23.83 & 22.17 & - & 26.27 & 26.26 & 25.67 & 23.37 & - \\
\hline 302-N100-FR & 4.00 & 93.86 & 93.80 & 93.20 & 85.10 & - & 104.53 & 104.29 & 102.32 & 94.27 & - \\
\hline 302-N100-FR & 6.00 & 196.88 & 195.50 & 187.10 & 169.77 & - & 230.30 & 229.11 & 203.98 & 194.41 & - \\
\hline 302-N120-FR & 1.98 & 26.01 & 25.97 & 25.30 & 22.38 & - & 27.17 & 27.16 & 27.12 & 23.49 & - \\
\hline 302-N120-FR & 4.00 & 97.47 & 97.47 & 95.87 & 86.44 & - & 111.32 & 111.12 & 110.55 & 97.50 & - \\
\hline 302-N120-FR & 6.00 & 202.58 & 201.16 & 193.42 & 174.59 & - & 241.24 & 239.93 & 233.04 & 199.24 & - \\
\hline 302-N150-FR & 1.99 & 27.71 & 27.51 & 26.10 & 23.24 & - & 28.56 & 28.55 & 28.54 & 24.25 & - \\
\hline 302-N150-FR & 4.00 & 103.08 & 102.77 & 98.41 & 89.34 & - & 120.82 & 120.58 & 118.63 & 101.37 & - \\
\hline 302-N150-FR & 6.00 & 210.15 & 208.63 & 199.80 & 179.71 & - & 247.08 & 246.04 & 240.85 & 205.67 & - \\
\hline
\end{tabular}


(b) $a / h$ for offset circular web opening case

\begin{tabular}{|c|c|c|c|c|c|c|c|c|c|}
\hline \multirow[t]{2}{*}{ Specimen } & \multirow{2}{*}{$\begin{array}{c}\text { Thickness } \\
t \\
(\mathrm{~mm})\end{array}$} & \multicolumn{4}{|c|}{ Unfastened FEA load per web, $\mathrm{P}_{\mathrm{FEA}}$} & \multicolumn{4}{|c|}{ Fastened FEA load per web, $\mathrm{P}_{\text {FEA }}$} \\
\hline & & $\begin{array}{l}A(0) \\
(k N)\end{array}$ & $\begin{array}{c}A(0.2) \\
(k N)\end{array}$ & $\begin{array}{c}A(0.4) \\
(k N)\end{array}$ & $\begin{array}{c}A(0.6) \\
(k N)\end{array}$ & $\begin{array}{l}A(0) \\
(k N)\end{array}$ & $\begin{array}{c}A(0.2) \\
(k N)\end{array}$ & $\begin{array}{c}A(0.4) \\
(k N)\end{array}$ & $\begin{array}{c}A(0.6) \\
(k N)\end{array}$ \\
\hline 142-N100-FR & 1.27 & 11.50 & 11.46 & 11.27 & 10.95 & 12.73 & 12.71 & 12.63 & 12.53 \\
\hline 142-N100-FR & 4.00 & 93.24 & 93.15 & 92.67 & 91.75 & 112.63 & 112.62 & 112.55 & 112.49 \\
\hline 142-N100-FR & 6.00 & 166.59 & 166.08 & 164.37 & 160.39 & 201.87 & 201.39 & 199.44 & 186.56 \\
\hline 142-N120-FR & 1.27 & 12.19 & 12.11 & 11.90 & 11.41 & 13.45 & 13.44 & 13.43 & 13.38 \\
\hline 142-N120-FR & 4.00 & 97.47 & 97.33 & 96.72 & 95.53 & 120.57 & 120.57 & 120.47 & 120.24 \\
\hline 142-N120-FR & 6.00 & 166.56 & 166.09 & 164.36 & 159.70 & 201.42 & 200.93 & 198.95 & 186.40 \\
\hline 142-N150-FR & 1.28 & 12.97 & 12.87 & 12.53 & 11.88 & 14.39 & 14.38 & 14.34 & 14.27 \\
\hline 142-N150-FR & 4.00 & 97.77 & 97.39 & 96.13 & 93.66 & 128.76 & 128.73 & 128.48 & 125.80 \\
\hline 142-N150-FR & 6.00 & 158.21 & 157.58 & 155.68 & 152.08 & 197.41 & 196.97 & 195.04 & 184.86 \\
\hline 202-N100-FR & 1.39 & 12.36 & 12.07 & 11.44 & 10.37 & 14.27 & 14.27 & 14.17 & 14.03 \\
\hline 202-N100-FR & 4.00 & 93.01 & 92.74 & 91.86 & 90.03 & 108.50 & 108.47 & 108.37 & 108.25 \\
\hline 202-N100-FR & 6.00 & 184.32 & 183.61 & 181.12 & 175.45 & 227.47 & 226.95 & 224.65 & 212.61 \\
\hline 202-N120-FR & 1.39 & 12.61 & 12.35 & 11.65 & 10.51 & 14.34 & 14.34 & 14.24 & 14.06 \\
\hline 202-N120-FR & 4.00 & 97.07 & 96.78 & 95.72 & 93.16 & 116.44 & 116.41 & 116.30 & 116.13 \\
\hline 202-N120-FR & 6.00 & 185.87 & 185.13 & 182.63 & 176.05 & 230.69 & 229.99 & 227.02 & 213.26 \\
\hline 202-N150-FR & 1.39 & 12.95 & 12.67 & 11.93 & 10.90 & 16.45 & 16.44 & 16.30 & 16.05 \\
\hline 202-N150-FR & 4.00 & 102.38 & 101.94 & 100.36 & 95.45 & 127.48 & 127.41 & 127.27 & 126.92 \\
\hline 202-N150-FR & 6.00 & 187.98 & 187.16 & 184.31 & 176.30 & 229.87 & 229.12 & 225.97 & 212.56 \\
\hline 302-N100-FR & 1.98 & 22.75 & 22.66 & 22.29 & 21.36 & 26.27 & 26.26 & 26.15 & 25.72 \\
\hline 302-N100-FR & 2.00 & 93.88 & 93.48 & 91.97 & 88.40 & 104.53 & 104.52 & 104.46 & 104.31 \\
\hline 302-N100-FR & 4.00 & 194.66 & 193.59 & 189.95 & 178.72 & 230.30 & 230.06 & 228.73 & 212.81 \\
\hline 302-N120-FR & 1.98 & 24.06 & 23.91 & 23.10 & 21.95 & 27.17 & 27.15 & 26.96 & 26.57 \\
\hline 302-N120-FR & 2.00 & 97.50 & 96.93 & 95.23 & 90.39 & 111.36 & 111.35 & 111.21 & 111.02 \\
\hline 302-N120-FR & 4.00 & 197.85 & 196.83 & 192.58 & 176.63 & 241.24 & 240.59 & 237.63 & 226.42 \\
\hline 302-N150-FR & 1.99 & 25.42 & 25.08 & 24.17 & 22.58 & 28.56 & 28.53 & 28.38 & 27.19 \\
\hline 302-N150-FR & 2.00 & 103.09 & 102.44 & 100.01 & 93.97 & 120.82 & 120.80 & 120.74 & 120.02 \\
\hline 302-N150-FR & 4.00 & 202.77 & 201.35 & 195.45 & 184.86 & 247.08 & 246.05 & 241.93 & 227.42 \\
\hline
\end{tabular}


(c) $x / h$ for offset circular web opening case

\begin{tabular}{|c|c|c|c|c|c|c|c|c|c|}
\hline \multirow[t]{2}{*}{ Specimen } & \multirow{2}{*}{$\begin{array}{c}\text { Thickness } \\
t \\
(\mathrm{~mm})\end{array}$} & \multicolumn{4}{|c|}{ Unfastened FEA load per web, $P(F E A)$} & \multicolumn{4}{|c|}{ Fastened FEA load per web, $\mathrm{P}_{\mathrm{FEA}}$} \\
\hline & & $\begin{array}{l}X(0) \\
(k N)\end{array}$ & $\begin{array}{c}X(0.2) \\
(k N)\end{array}$ & $\begin{array}{c}X(0.4) \\
(k N)\end{array}$ & $\begin{array}{c}X(0.6) \\
(k N)\end{array}$ & $\begin{array}{l}X(0) \\
(k N)\end{array}$ & $\begin{array}{c}X(0.2) \\
(k N)\end{array}$ & $\begin{array}{c}X(0.4) \\
(k N)\end{array}$ & $\begin{array}{c}X(0.6) \\
(k N)\end{array}$ \\
\hline 142-N100-A0-FR & 1.27 & 11.13 & 11.13 & 11.13 & 11.13 & 12.12 & 12.12 & 12.12 & 12.12 \\
\hline $142-\mathrm{N} 100-\mathrm{A} 0.2-\mathrm{FR}$ & 1.27 & 10.99 & 10.99 & 11.00 & 11.03 & 12.04 & 12.05 & 12.06 & 12.08 \\
\hline 142-N100-A0.4-FR & 1.27 & 10.50 & 10.54 & 10.64 & 10.79 & 11.75 & 11.80 & 11.87 & 11.99 \\
\hline 142-N100-A0.6-FR & 1.27 & 9.56 & 9.80 & 10.12 & 10.44 & 11.09 & 11.26 & 11.60 & 11.88 \\
\hline 142-N100-A0.8-FR & 1.27 & 8.15 & 8.76 & 9.21 & 9.60 & --- & --- & --- & --- \\
\hline 142-N120-A0-FR & 1.27 & 11.88 & 11.88 & 11.88 & 11.88 & 12.99 & 12.99 & 12.99 & 12.99 \\
\hline $142-\mathrm{N} 120-\mathrm{A} 0.2-\mathrm{FR}$ & 1.27 & 11.70 & 11.71 & 11.72 & 11.76 & 12.91 & 12.92 & 12.93 & 12.96 \\
\hline 142-N120-A0.4-FR & 1.27 & 11.07 & 11.09 & 11.26 & 11.45 & 12.56 & 12.56 & 12.73 & 12.88 \\
\hline 142-N120-A0.6-FR & 1.27 & 9.85 & 10.16 & 10.52 & 10.86 & 11.66 & 11.95 & 12.40 & 12.74 \\
\hline 142-N120-A0.8-FR & 1.27 & 8.43 & 9.05 & 9.53 & 9.85 & 9.55 & 10.74 & 11.64 & 12.38 \\
\hline 142-N150-A0-FR & 1.28 & 12.75 & 12.75 & 12.75 & 12.75 & 14.42 & 14.42 & 14.42 & 14.42 \\
\hline 142-N150-A0.2-FR & 1.28 & 12.56 & 12.58 & 12.60 & 12.62 & 13.87 & 13.90 & 13.91 & 14.34 \\
\hline 142-N150-A0.4-FR & 1.28 & 11.64 & 11.70 & 11.91 & 12.31 & 13.35 & 13.40 & 13.62 & 13.81 \\
\hline 142-N150-A0.6-FR & 1.28 & 10.35 & 10.68 & 11.04 & 11.37 & 12.26 & 12.66 & 13.16 & 13.58 \\
\hline 142-N150-A0.8-FR & 1.28 & 8.96 & 9.53 & 9.98 & 10.27 & 10.29 & 11.36 & 12.21 & 12.98 \\
\hline 202-N100-A0-FR & 1.39 & 12.40 & 12.40 & 12.40 & 12.40 & 13.50 & 13.50 & 13.50 & 13.50 \\
\hline 202-N100-A0.2-FR & 1.39 & 12.10 & 12.11 & 12.12 & 12.14 & 13.39 & 13.40 & 13.41 & 13.47 \\
\hline 202-N100-A0.4-FR & 1.39 & 10.98 & 11.06 & 11.21 & 11.41 & 12.26 & 12.27 & 12.44 & 12.67 \\
\hline 202-N100-A0.6-FR & 1.39 & 9.46 & 9.76 & 10.08 & 10.36 & 11.28 & 11.53 & 11.98 & 12.44 \\
\hline 202-N120-A0-FR & 1.39 & 12.69 & 12.69 & 12.69 & 12.69 & 15.16 & 15.16 & 15.16 & 15.16 \\
\hline 202-N120-A0.2-FR & 1.39 & 12.35 & 12.37 & 12.38 & 12.39 & 15.05 & 15.05 & 15.07 & 15.14 \\
\hline 202-N120-A0.4-FR & 1.39 & 11.19 & 11.41 & 11.44 & 11.63 & 14.30 & 14.35 & 14.63 & 14.90 \\
\hline 202-N120-A0.6-FR & 1.39 & 9.66 & 9.96 & 10.26 & 10.53 & 13.00 & 13.38 & 13.95 & 14.54 \\
\hline 202-N150-A0-FR & 1.45 & 14.28 & 14.28 & 14.28 & 14.28 & 16.45 & 16.45 & 16.45 & 16.45 \\
\hline 202-N150-A0.2-FR & 1.45 & 13.87 & 13.88 & 13.89 & 13.94 & 16.26 & 16.27 & 16.30 & 16.38 \\
\hline 202-N150-A0.4-FR & 1.45 & 12.61 & 12.71 & 12.88 & 13.06 & 15.28 & 15.38 & 15.71 & 16.04 \\
\hline 202-N150-A0.6-FR & 1.45 & 11.00 & 11.30 & 11.60 & 11.85 & 13.78 & 14.23 & 14.87 & 15.52 \\
\hline 302-N100-A0-FR & 1.98 & 24.25 & 24.25 & 24.25 & 24.25 & 25.62 & 25.62 & 25.62 & 25.62 \\
\hline $302-\mathrm{N} 100-\mathrm{A} 0.2-\mathrm{FR}$ & 1.98 & 24.00 & 24.01 & 24.03 & 24.09 & 26.55 & 26.56 & 25.60 & 25.58 \\
\hline 302-N120-A0-FR & 1.96 & 25.23 & 25.23 & 25.23 & 25.23 & 26.63 & 26.63 & 26.63 & 26.63 \\
\hline 302-N120-A0.2-FR & 1.96 & 24.80 & 24.83 & 24.86 & 24.94 & 26.51 & 26.53 & 26.62 & 26.59 \\
\hline 302-N120-A0.4-FR & 1.96 & 22.74 & 22.87 & 23.29 & 23.79 & 24.46 & 24.66 & 25.34 & 25.45 \\
\hline 302-N120-A0.6-FR & 1.96 & 18.80 & 20.00 & 21.35 & 23.24 & 23.25 & 23.30 & 24.50 & 24.59 \\
\hline 302-N150-A0-FR & 1.99 & 27.55 & 27.55 & 27.55 & 27.55 & 28.10 & 28.10 & 28.10 & 28.10 \\
\hline 302-N150-A0.2-FR & 1.99 & 26.79 & 26.83 & 26.87 & 27.02 & 27.93 & 28.02 & 28.10 & 28.41 \\
\hline 302-N150-A0.4-FR & 1.99 & 24.26 & 24.40 & 24.87 & 25.44 & 26.90 & 27.25 & 27.63 & 27.83 \\
\hline 302-N150-A0.6-FR & 1.99 & 20.84 & 21.56 & 22.58 & 23.74 & 23.84 & 24.86 & 26.59 & 26.70 \\
\hline
\end{tabular}


Table 4 Coefficients of the proposed strength reduction factor equations

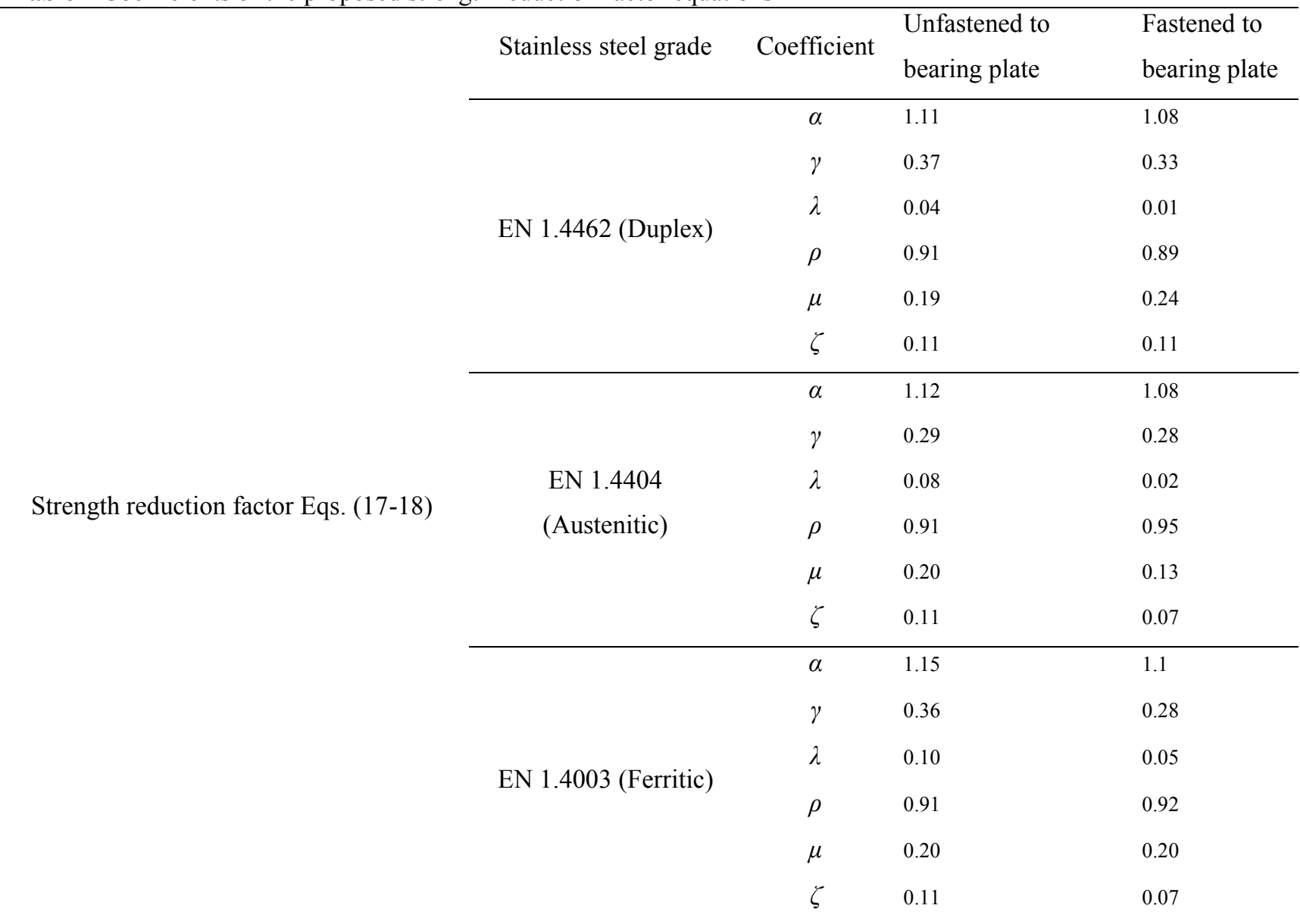


Table 5 Comparison of web crippling strength reduction factor for cold-formed stainless steel lipped channel-sections with reduction factors equations proposed by Lian et al. (2016a,b,e)

(a) Flange unfastened to the bearing plate

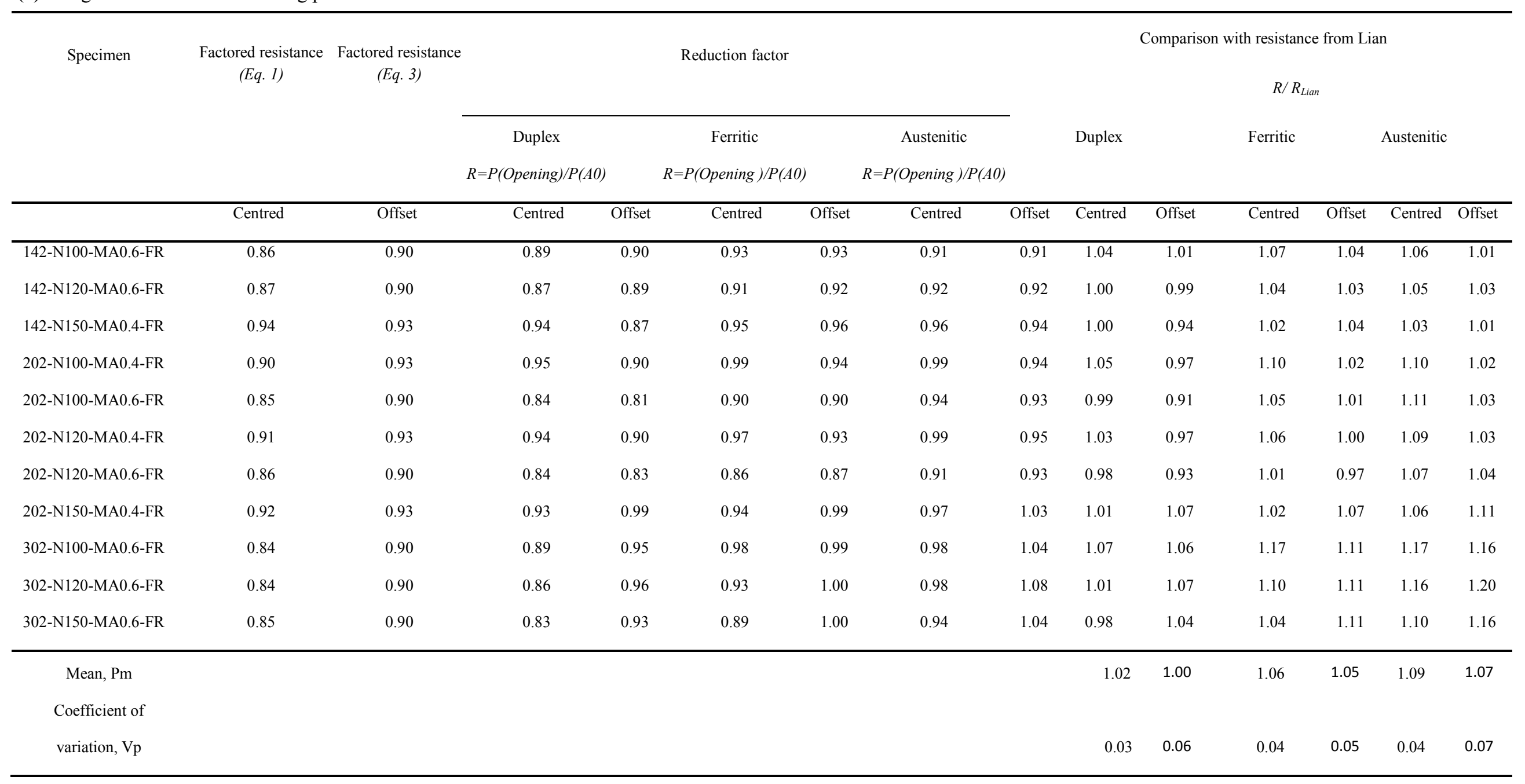


(b) Flange fastened to the bearing plate

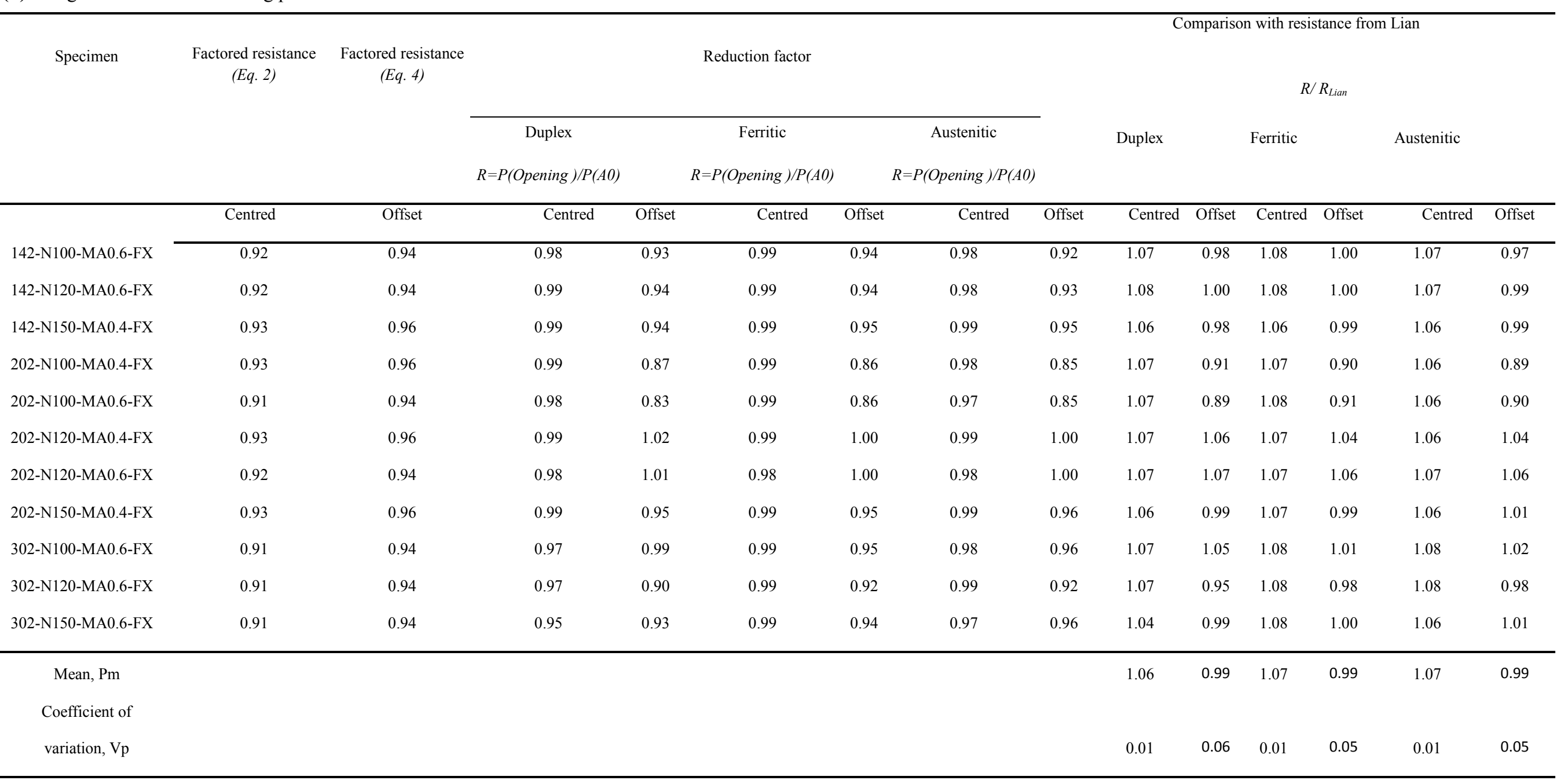


Table 6 Statistical analysis of strength reduction factor

(a) Duplex stainless steel grade

\begin{tabular}{ccccc}
\hline \multirow{2}{*}{ Statistical parameters } & \multicolumn{2}{c}{$\begin{array}{c}\text { Centred circular web opening } \\
\left.R_{(F E A}\right) / R_{p}\end{array}$} & \multicolumn{2}{c}{$\begin{array}{c}\text { Offset circular web opening } \\
R_{(F E A)} / R_{p}\end{array}$} \\
\cline { 2 - 5 } & $\begin{array}{c}\text { Unfastened } \\
\text { to bearing plate }\end{array}$ & $\begin{array}{c}\text { Fastened } \\
\text { to bearing plate }\end{array}$ & $\begin{array}{c}\text { Unfastened } \\
\text { to bearing plate }\end{array}$ & $\begin{array}{c}\text { Fastened } \\
\text { to bearing plate }\end{array}$ \\
\hline Number of data & 90 & 90 & 84 & 81 \\
Mean, $P_{m}$ & 1.00 & 1.01 & 1.04 & 1.04 \\
Coefficient of variation, $V_{p}$ & 0.03 & 0.03 & 0.04 & 0.05 \\
Reliability index, $\beta$ & 2.82 & 2.86 & 2.97 & 2.94 \\
Resistance factor, $\phi$ & 0.85 & 0.85 & 0.85 & 0.85 \\
\hline
\end{tabular}


(b) Austenitic stainless steel grade

\begin{tabular}{ccccc}
\hline \multirow{2}{*}{ Statistical parameters } & \multicolumn{2}{c}{$\begin{array}{c}\text { Centred circular web opening } \\
R_{(F E A)} / R_{p}\end{array}$} & \multicolumn{2}{c}{$\begin{array}{c}\text { Offset circular web opening } \\
R_{(F E A)} / R_{p}\end{array}$} \\
\cline { 2 - 5 } & $\begin{array}{c}\text { Unfastened } \\
\text { to bearing plate }\end{array}$ & $\begin{array}{c}\text { Fastened } \\
\text { to bearing plate }\end{array}$ & $\begin{array}{c}\text { Unfastened } \\
\text { to bearing plate }\end{array}$ & $\begin{array}{c}\text { Fastened } \\
\text { to bearing plate }\end{array}$ \\
\hline Number of data & 90 & 90 & 84 & 81 \\
Mean, $P_{m}$ & 0.99 & 0.99 & 1.00 & 1.01 \\
Coefficient of variation, $V_{p}$ & 0.05 & 0.03 & 0.03 & 0.03 \\
Reliability index, $\beta$ & 2.74 & 2.78 & 2.82 & 2.86 \\
Resistance factor, $\phi$ & 0.85 & 0.85 & 0.85 & 0.85 \\
\hline
\end{tabular}


(c) Ferritic stainless steel grade

\begin{tabular}{ccccc}
\hline \multirow{2}{*}{ Statistical parameters } & \multicolumn{2}{c}{$\begin{array}{c}\text { Centred circular web opening } \\
R_{(F E A)} / R_{p}\end{array}$} & \multicolumn{2}{c}{$\begin{array}{c}\text { Offset circular web opening } \\
R_{(F E A)} / R_{p}\end{array}$} \\
\cline { 2 - 5 } & $\begin{array}{c}\text { Unfastened } \\
\text { to bearing plate }\end{array}$ & $\begin{array}{c}\text { Fastened } \\
\text { to bearing plate }\end{array}$ & $\begin{array}{c}\text { Unfastened } \\
\text { to bearing plate }\end{array}$ & $\begin{array}{c}\text { Fastened } \\
\text { to bearing plate }\end{array}$ \\
\hline Number of data & 90 & 90 & 84 & 81 \\
Mean, $P_{m}$ & 1.00 & 0.99 & 1.00 & 1.0 \\
Coefficient of variation, $V_{p}$ & 0.05 & 0.03 & 0.02 & 0.03 \\
Reliability index, $\beta$ & 2.78 & 2.78 & 2.83 & 2.82 \\
Resistance factor, $\phi$ & 0.85 & 0.85 & 0.85 & 0.85 \\
\hline
\end{tabular}




\section{Figure Captions:}

Fig. 1 Photograph of cold-formed stainless steel lipped channel-sections with circular web openings after Lawson et. al. (2015)

Fig. 2 Interior-one-flange (IOF) loading condition after Lian et al. (2016a,b,e)

(a) With circular web opening centred under bearing plate

(b) With circular web openings offset from bearing plate

Fig. 3 Experimental analysis of cold-formed steel lipped channel-sections under interior-one-flange (IOF) loading condition for the case of flange unfastened to bearing plate after Lian et al. (2016a,b,e);

(a) Centred circular web opening

(b) Offset circular web opening

Fig. 4 Definition of symbols

Fig. 5 Finite element model of cold-formed steel lipped channel-sections under interior-one-flange (IOF) loading condition for the case of flange unfastened to bearing plate after Lian et al. $(2016 \mathrm{a}, \mathrm{b}, \mathrm{e})$;

(a) Centred circular web opening

(b) Offset circular web opening

Fig. 6 Comparison of finite element analysis and experimental results with Lian et al. (2016a,b,e)

(a) Centred circular web opening for the case of flange unfastened to bearing plate

(b) Offset circular web opening for the case of flange fastened to bearing plate

Fig. 7 Variation in reduction factors for $\mathrm{C} 142$ section for the case of flange unfastened to bearing plate

(a) with $a / h$ for centered circular web opening

(b) with $x / h$ for offset circular web opening

Fig. 8 Comparison of strength reduction factor for $\mathrm{C} 142$ section for the case of flange unfastened to bearing plate

(a) with centered circular web opening

(b) with offset circular web opening 


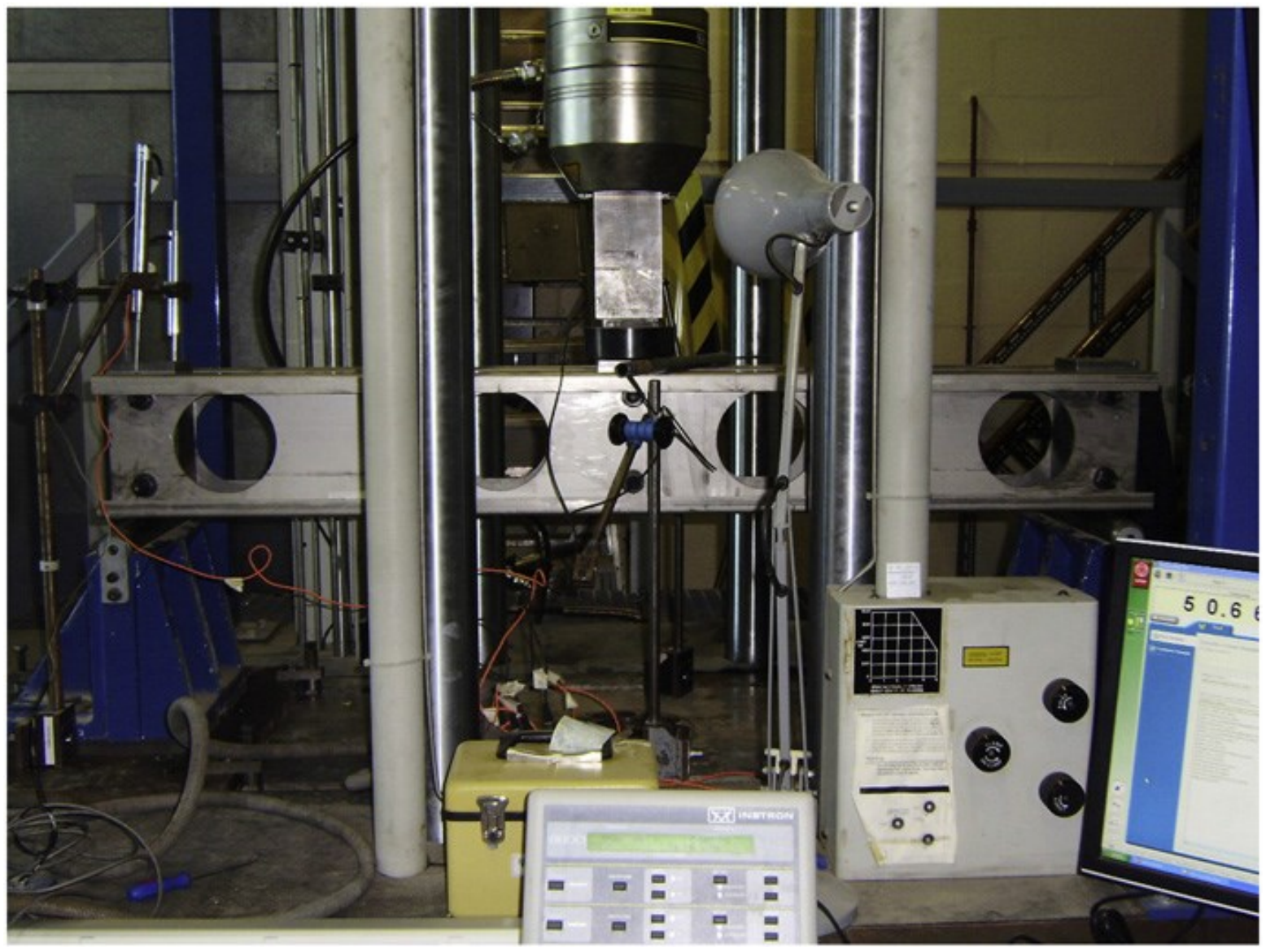

Fig. 1 Photograph of cold-formed stainless steel lipped channel-sections with circular web openings after Lawson et. al. (2015) 


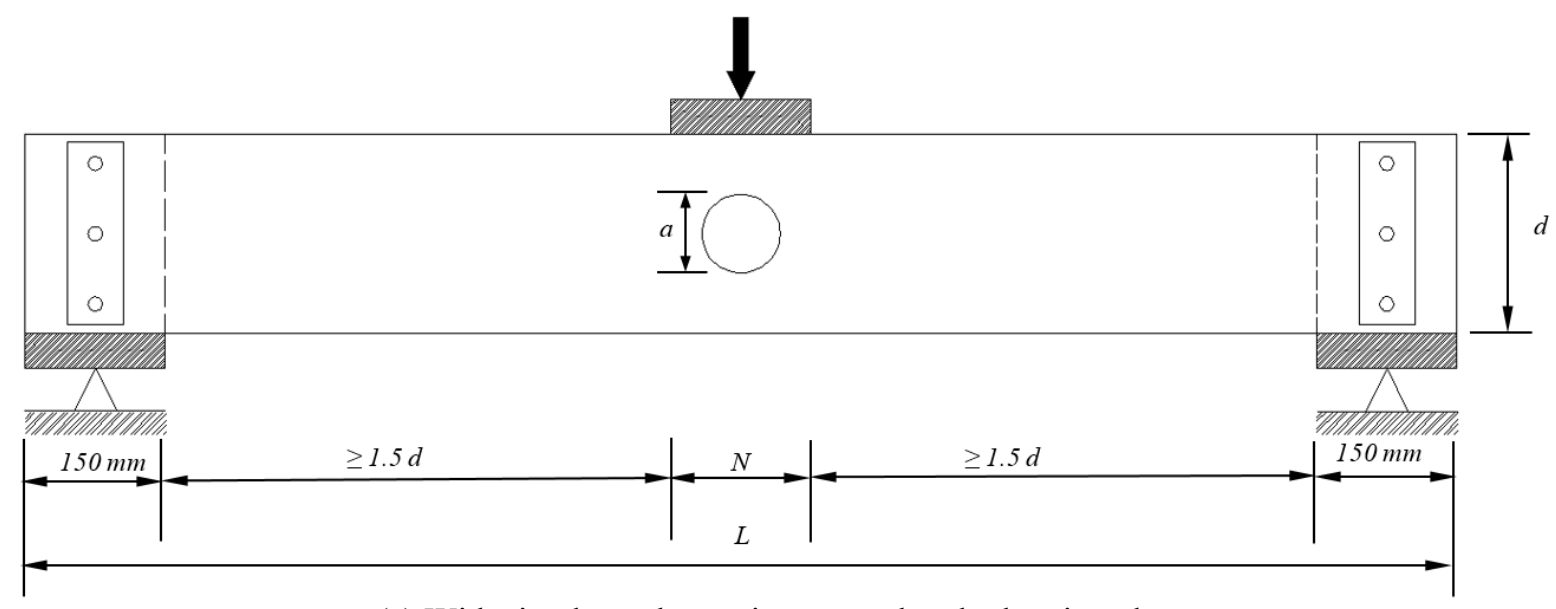

(a) With circular web opening centred under bearing plate

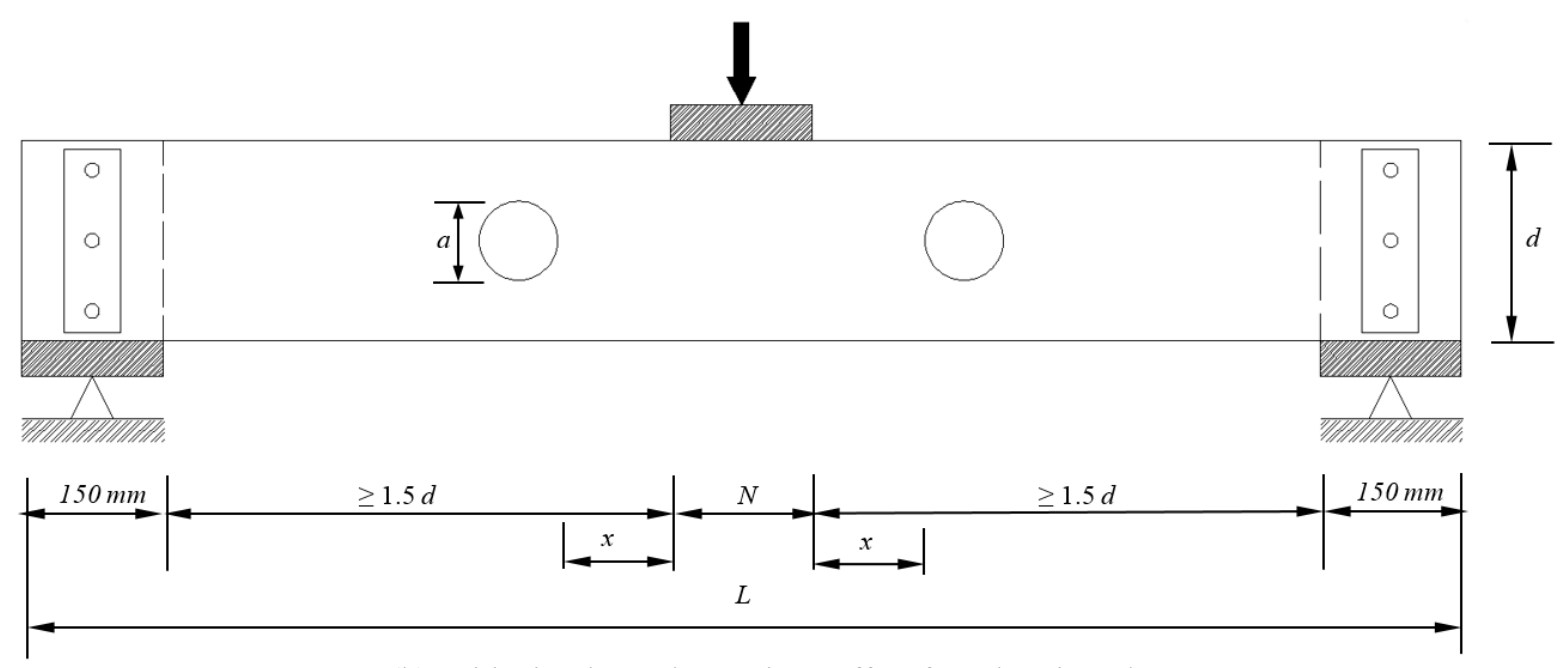

(b) With circular web openings offset from bearing plate

Fig. 2 Interior-one-flange (IOF) loading condition after Lian et al. (2016a,b,e) 


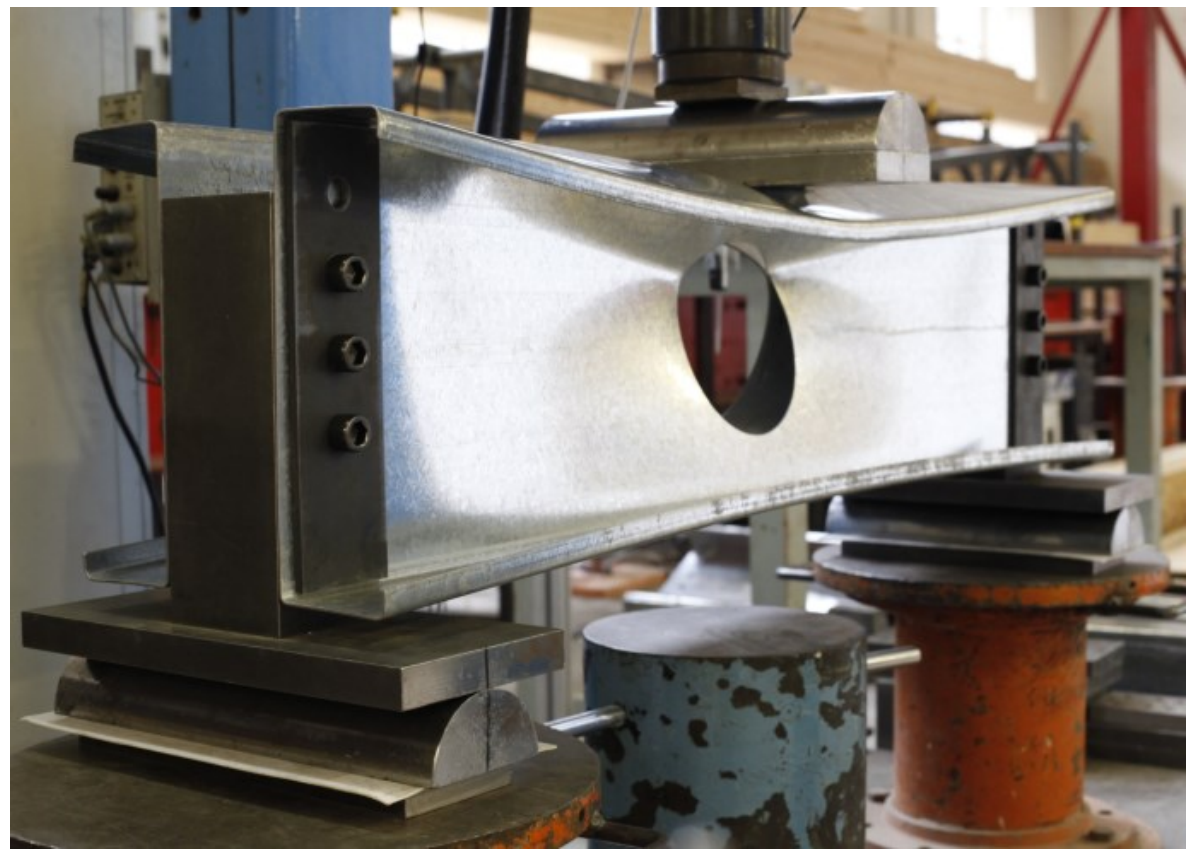

(a) Centred circular web opening

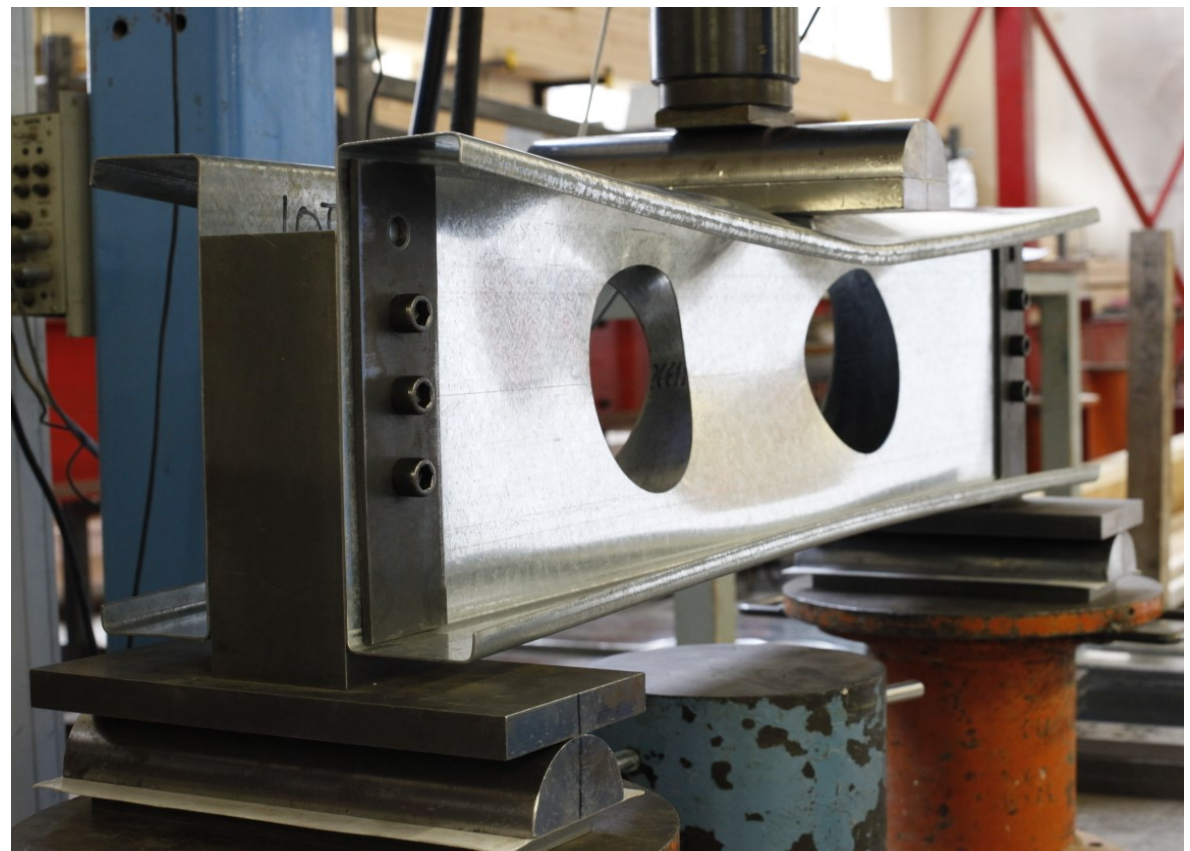

(b) Offset circular web opening

Fig. 3 Experimental analysis of cold-formed steel lipped channel-sections under interior-one-flange (IOF) loading condition for the case of flange unfastened to bearing plate after Lian et al. $(2016 \mathrm{a}, \mathrm{b}, \mathrm{e})$ 


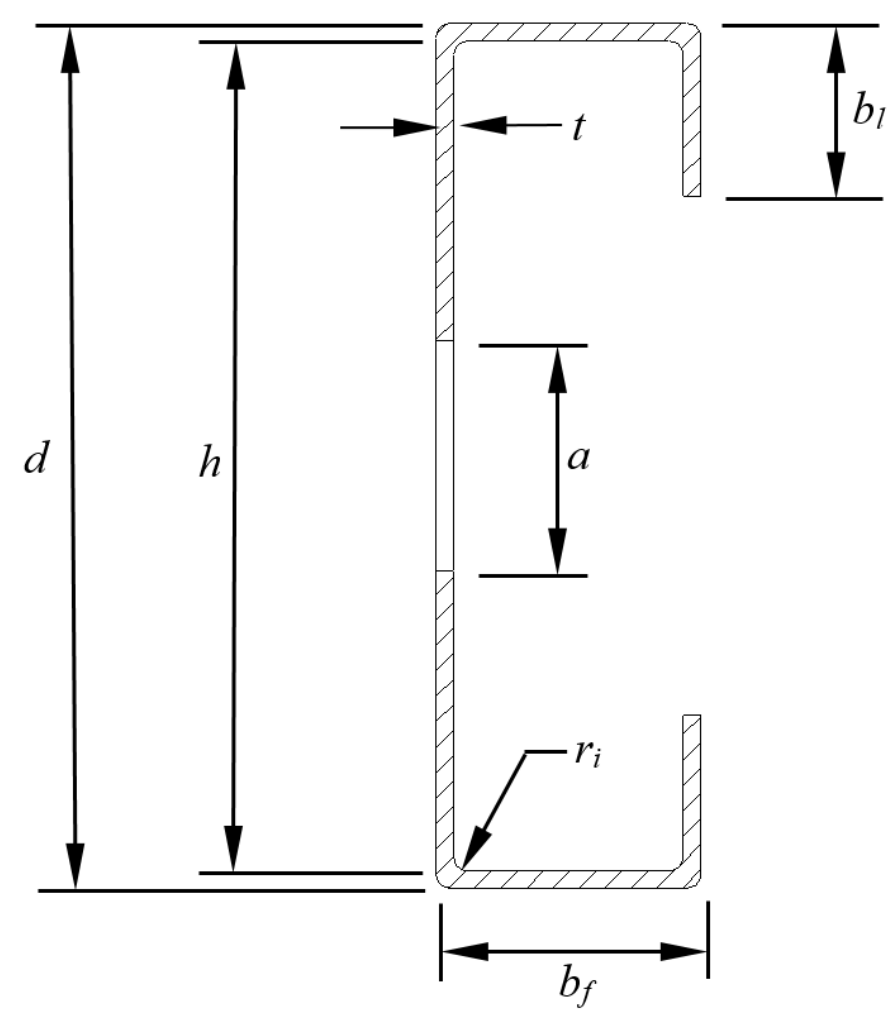

Fig. 4 Definition of symbols 


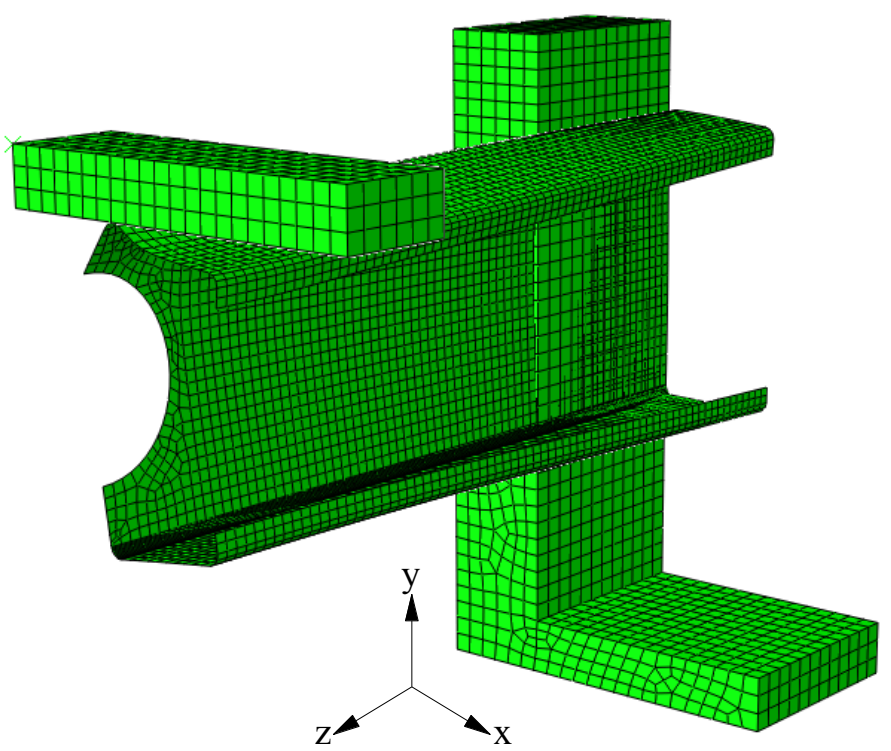

(a) Centred circular web opening

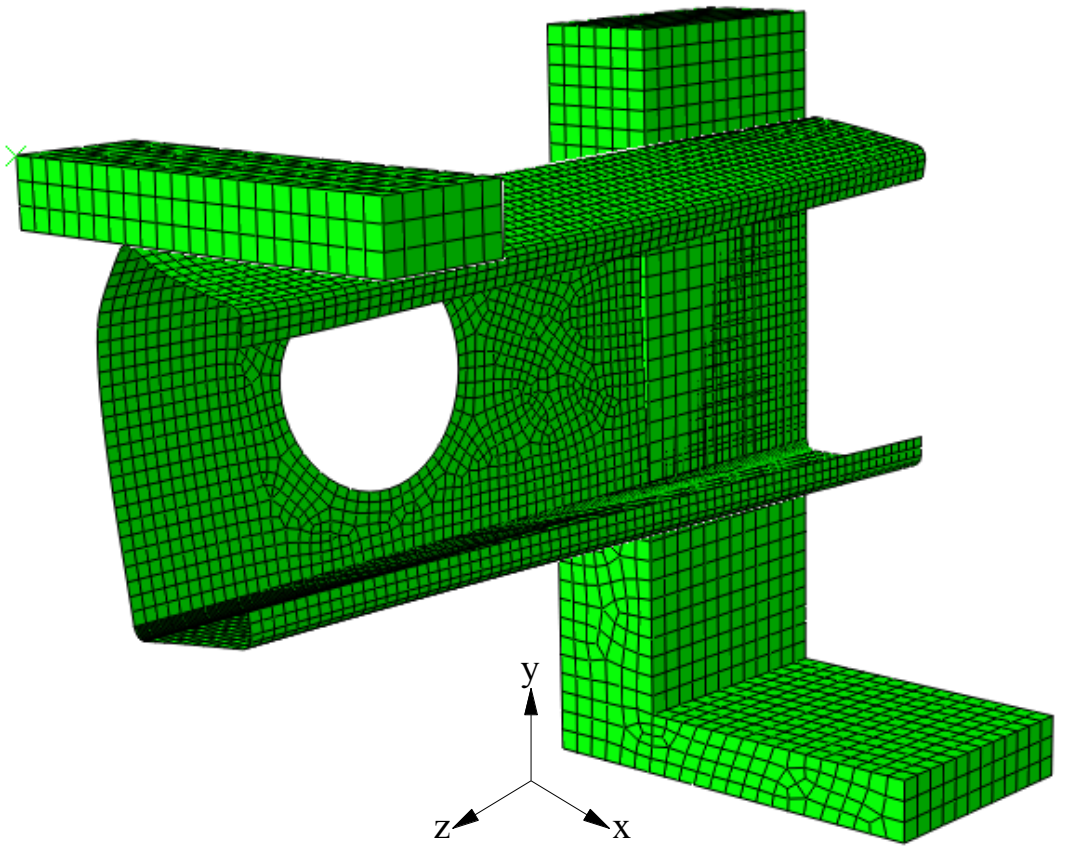

(b) Offset circular web opening

Fig. 5 Deformed shape predicted from finite element analysis of cold-formed steel lipped channel-sections under interior-one-flange (IOF) loading condition for case of flange unfastened to bearing plate after Lian et al. $(2016 \mathrm{a}, \mathrm{b}, \mathrm{e})$ 


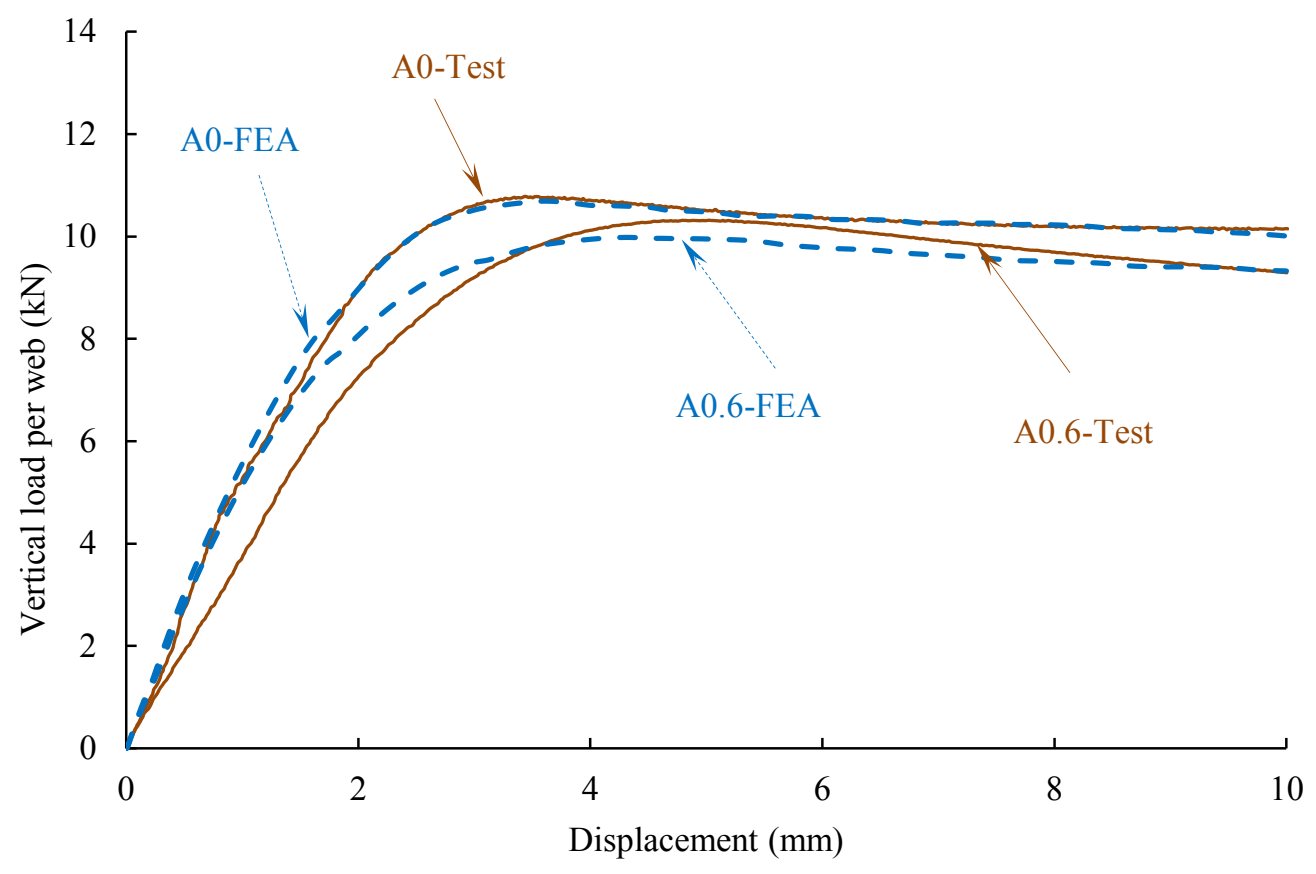

(a) Centred circular web opening for the case of flange unfastened to bearing plate

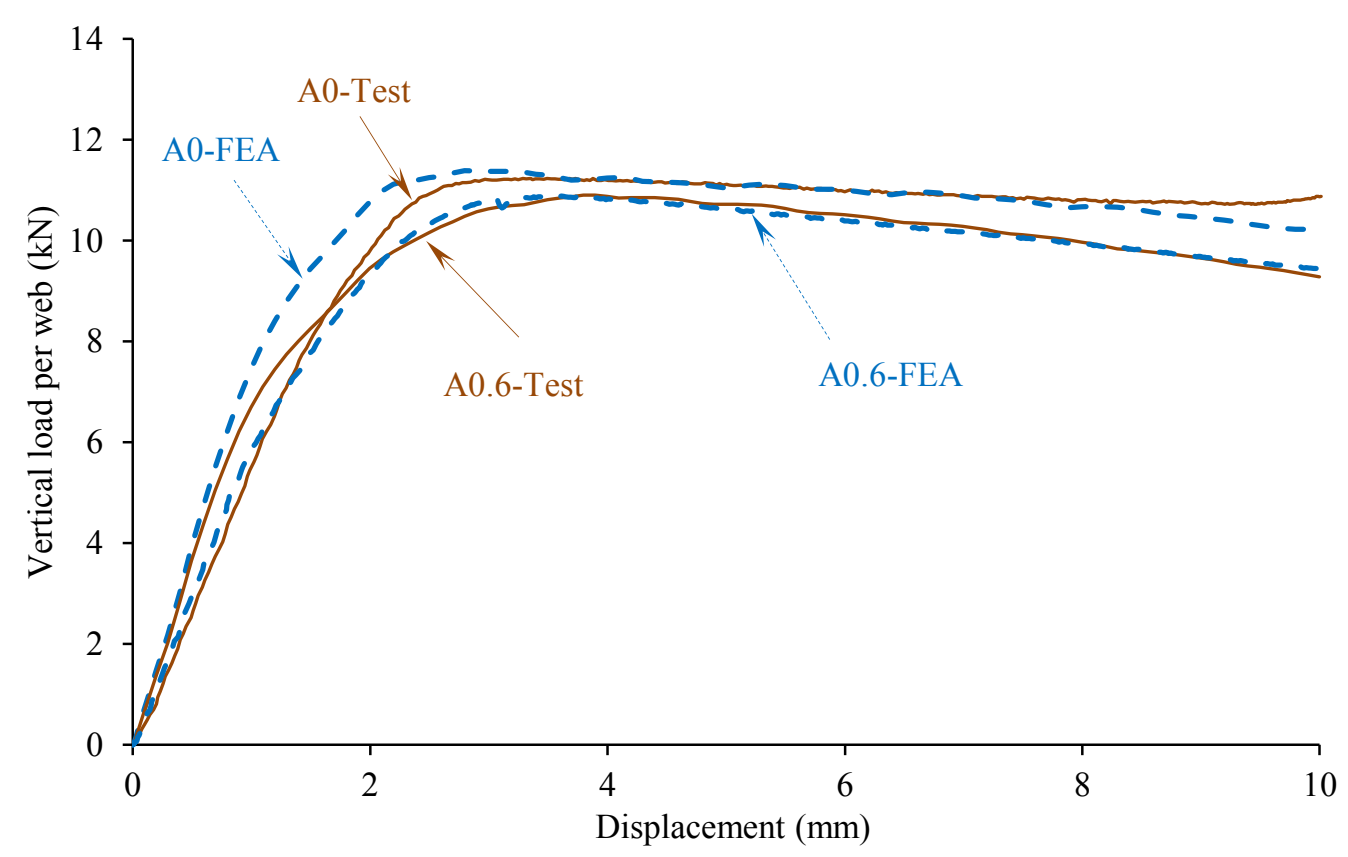

(b) Offset circular web opening for the case of flange fastened to bearing plate

Fig. 6 Comparison of finite element results and experimental test results (Lian et al. 2016a,b,e) 


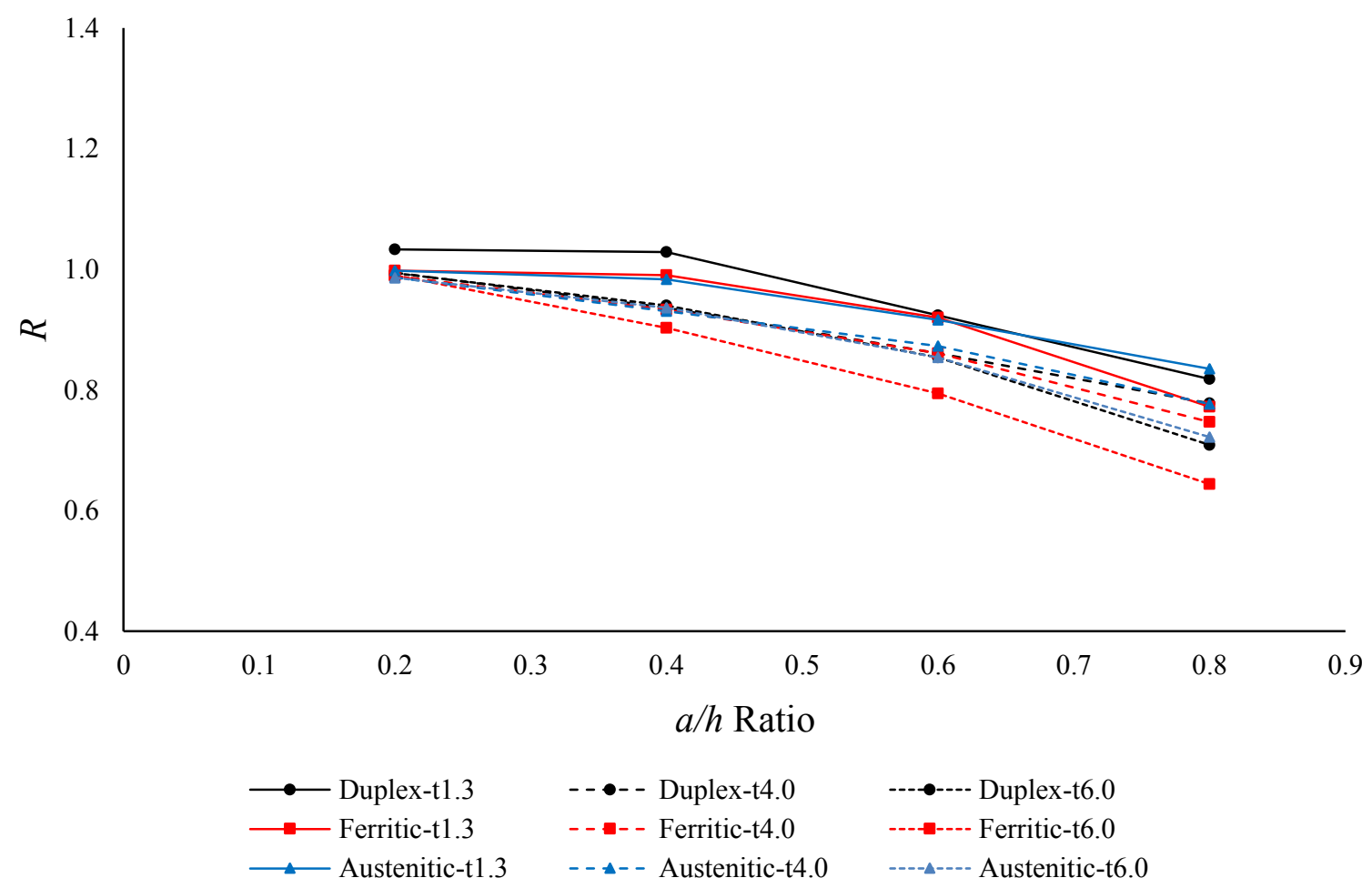

(a) with $a / h$ for centered circular web opening

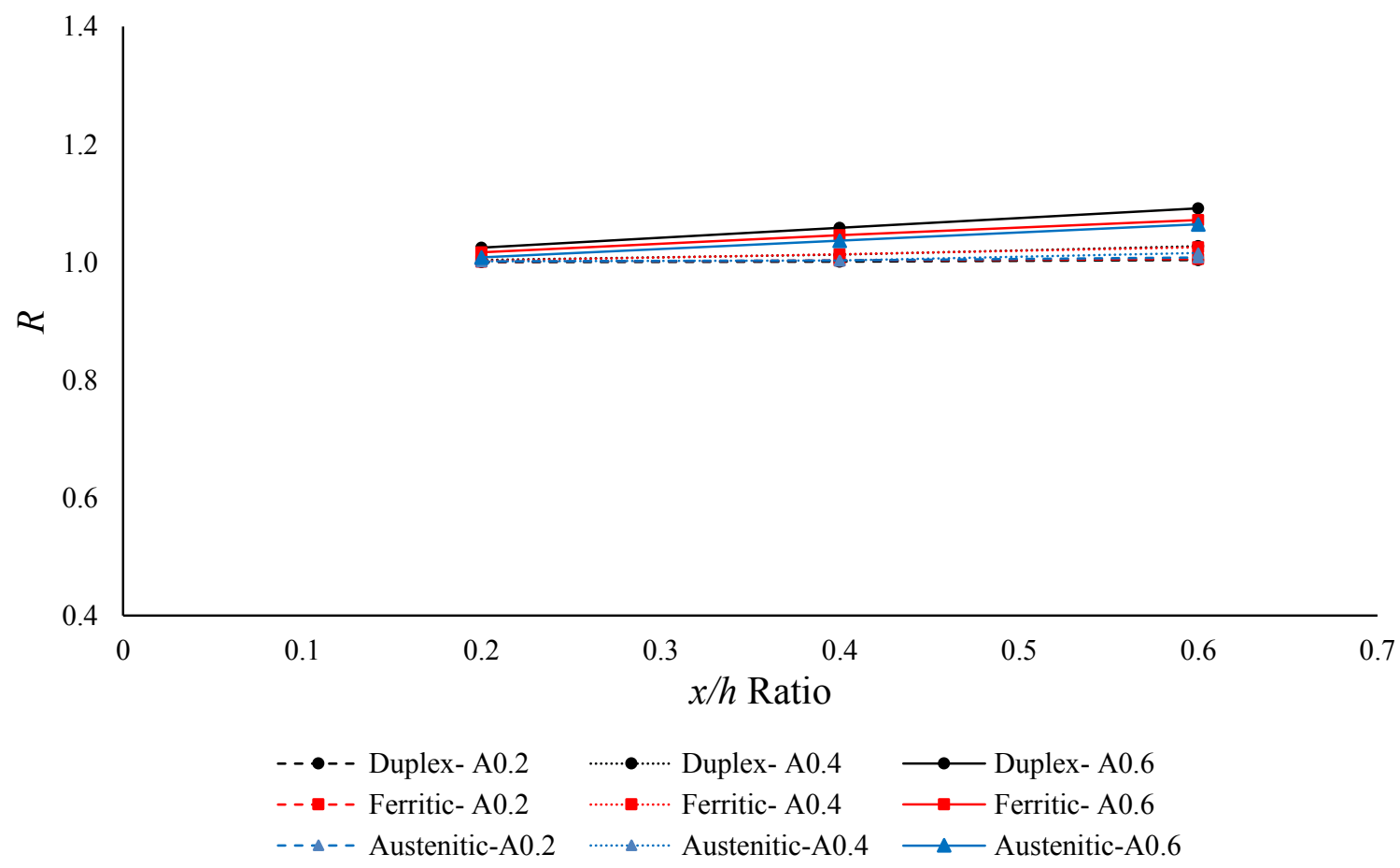

(b) with $x / h$ for offset circular web opening

Fig. 7 Variation in reduction factors for $\mathrm{C} 142$ section for the case of flange unfastened to bearing plate 


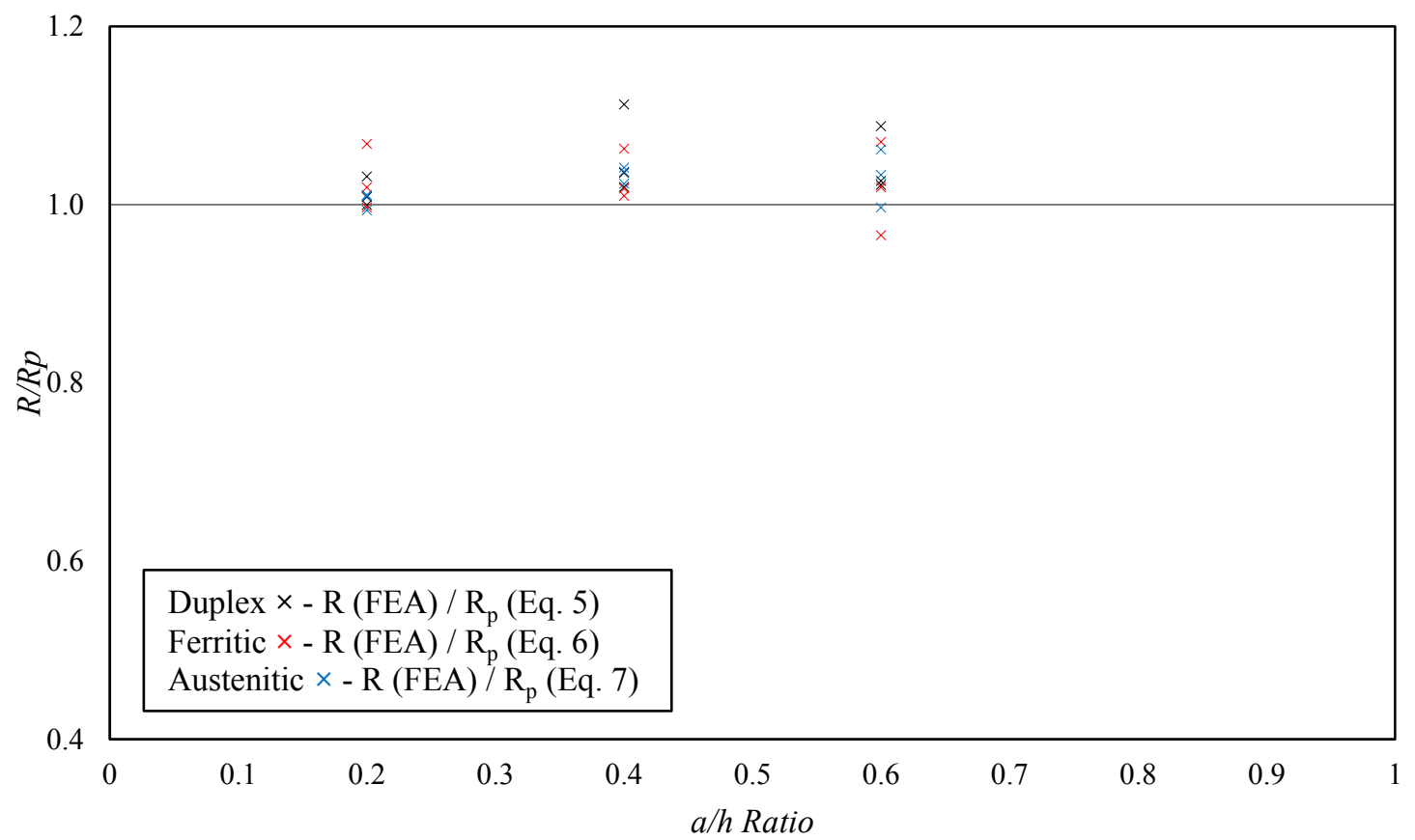

(a) with centered circular web opening

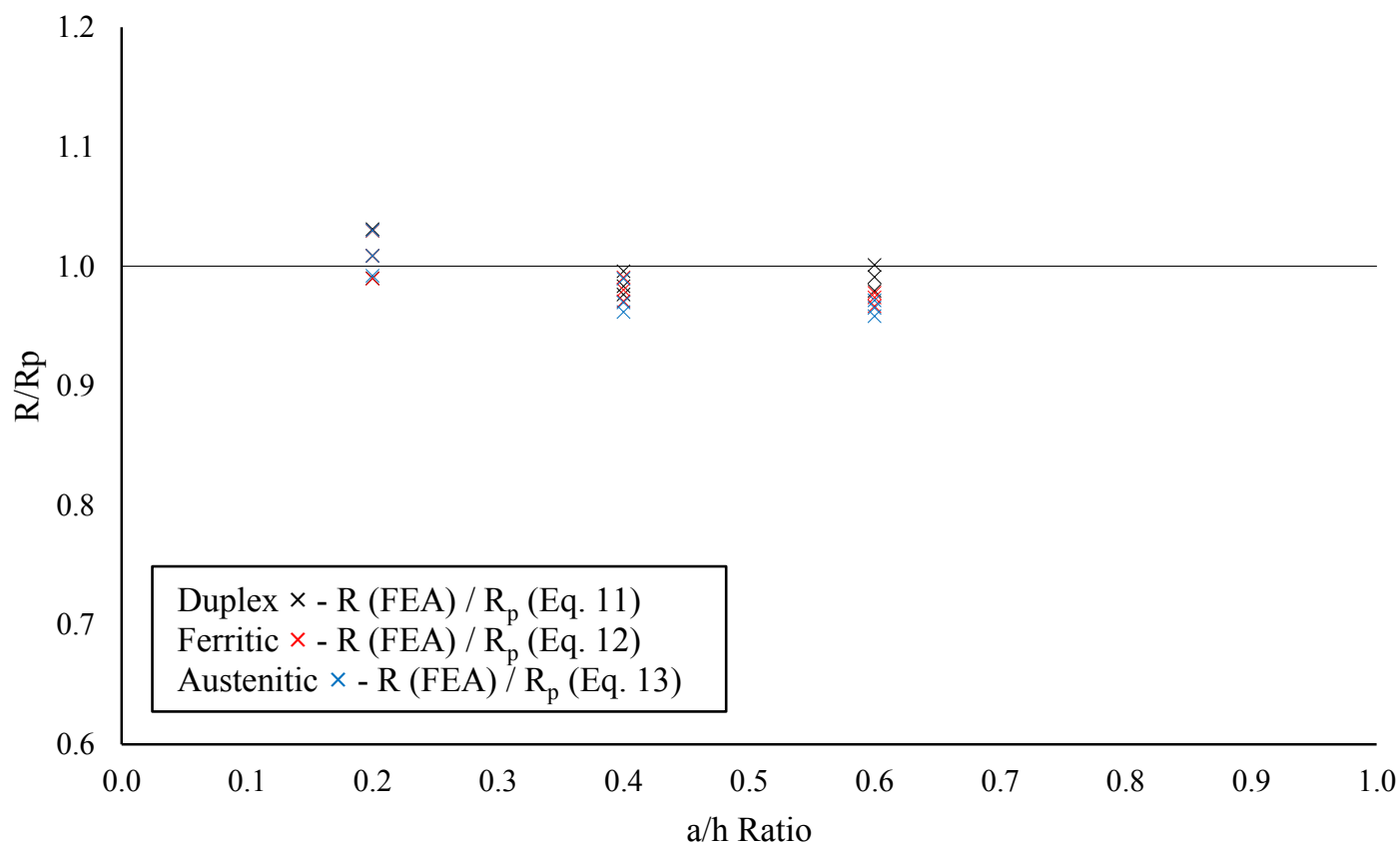

(b) with offset circular web opening

Fig. 8 Comparison of strength reduction factor for $\mathrm{C} 142$ section for the case of flange unfastened to bearing plate 


\section{Appendix A: Austenitic}

Table A1 Web crippling strengths of austenitic stainless steel sections predicted from finite element analysis (a) $a / h$ for centred circular web opening case

\begin{tabular}{|c|c|c|c|c|c|c|c|c|c|c|c|}
\hline \multirow[t]{2}{*}{ Specimen } & \multirow{2}{*}{$\begin{array}{c}\text { Thickness } \\
t \\
(\mathrm{~mm})\end{array}$} & \multicolumn{5}{|c|}{ Unfastened FEA load per web, $P_{\mathrm{FEA}}$} & \multicolumn{5}{|c|}{ Fastened FEA load per web, $P_{\text {FEA }}$} \\
\hline & & $\begin{array}{l}A(0) \\
(k N)\end{array}$ & $\begin{array}{c}A(0.2) \\
(k N)\end{array}$ & $\begin{array}{c}A(0.4) \\
(k N)\end{array}$ & $\begin{array}{c}A(0.6) \\
(k N)\end{array}$ & $\begin{array}{c}A(0.8) \\
(k N)\end{array}$ & $\begin{array}{l}A(0) \\
(k N)\end{array}$ & $\begin{array}{c}A(0.2) \\
(k N)\end{array}$ & $\begin{array}{c}A(0.4) \\
(k N)\end{array}$ & $\begin{array}{c}A(0.6) \\
(k N)\end{array}$ & $\begin{array}{c}A(0.8) \\
(k N)\end{array}$ \\
\hline 142-N100-FR & 1.27 & 8.52 & 8.50 & 8.38 & 7.81 & 7.12 & 9.40 & 9.38 & 9.31 & 8.64 & 7.95 \\
\hline 142-N100-FR & 4.00 & 66.53 & 65.63 & 61.92 & 58.06 & 51.69 & 80.79 & 80.28 & 76.54 & 69.72 & 62.78 \\
\hline 142-N100-FR & 6.00 & 124.69 & 122.98 & 116.66 & 106.49 & 90.00 & 155.17 & 153.62 & 147.49 & 136.90 & 124.44 \\
\hline 142-N120-FR & 1.27 & 8.90 & 8.88 & 8.85 & 8.21 & 7.46 & 9.86 & 9.84 & 9.82 & 9.04 & 8.07 \\
\hline 142-N120-FR & 4.00 & 69.46 & 68.40 & 65.29 & 61.55 & 52.84 & 86.15 & 85.73 & 81.50 & 73.13 & 67.20 \\
\hline 142-N120-FR & 6.00 & 123.59 & 121.87 & 115.61 & 103.50 & 87.08 & 155.65 & 154.14 & 148.69 & 139.17 & 123.82 \\
\hline 142-N150-FR & 1.28 & 9.61 & 9.60 & 9.31 & 8.57 & 7.94 & 10.70 & 10.65 & 10.46 & 9.63 & 8.71 \\
\hline 142-N150-FR & 4.00 & 69.85 & 69.06 & 66.42 & 59.16 & 49.80 & 91.06 & 90.57 & 87.07 & 81.59 & 73.54 \\
\hline 142-N150-FR & 6.00 & 116.53 & 114.51 & 108.13 & 96.77 & 80.78 & 152.49 & 151.56 & 147.56 & 139.37 & 128.29 \\
\hline 202-N100-FR & 1.39 & 9.60 & 9.58 & 9.57 & 9.07 & - & 10.08 & 10.05 & 10.01 & 9.88 & - \\
\hline 202-N100-FR & 4.00 & 67.78 & 67.00 & 64.06 & 57.82 & - & 78.76 & 78.15 & 75.81 & 67.73 & - \\
\hline 202-N100-FR & 6.00 & 136.21 & 133.39 & 125.44 & 114.45 & - & 167.89 & 166.07 & 159.14 & 142.91 & - \\
\hline 202-N120-FR & 1.39 & 10.15 & 10.13 & 10.10 & 9.31 & - & 11.20 & 11.15 & 10.97 & 10.71 & - \\
\hline 202-N120-FR & 4.00 & 70.62 & 69.85 & 66.26 & 60.38 & - & 84.52 & 83.83 & 80.22 & 72.09 & - \\
\hline 202-N120-FR & 6.00 & 138.79 & 136.21 & 128.95 & 117.15 & - & 177.96 & 177.04 & 168.09 & 151.94 & - \\
\hline 202-N150-FR & 1.39 & 10.90 & 10.91 & 10.65 & 9.70 & - & 12.23 & 12.15 & 11.58 & 11.29 & - \\
\hline 202-N150-FR & 4.00 & 74.61 & 73.64 & 69.65 & 64.74 & - & 93.02 & 91.72 & 86.48 & 78.87 & - \\
\hline 202-N150-FR & 6.00 & 139.73 & 137.25 & 130.06 & 117.07 & - & 189.91 & 182.93 & 175.42 & 161.41 & - \\
\hline 302-N100-FR & 1.98 & 18.40 & 18.36 & 18.22 & 18.19 & - & 19.25 & 19.20 & 18.48 & 17.93 & - \\
\hline 302-N100-FR & 4.00 & 69.18 & 69.90 & 63.73 & 63.16 & - & 76.33 & 75.92 & 74.43 & 69.63 & - \\
\hline 302-N100-FR & 6.00 & 146.79 & 144.60 & 121.18 & 123.08 & - & 168.71 & 167.10 & 160.52 & 147.76 & - \\
\hline 302-N120-FR & 1.98 & 19.41 & 19.39 & 19.30 & 19.12 & - & 19.75 & 19.71 & 19.68 & 18.59 & - \\
\hline 302-N120-FR & 4.00 & 72.56 & 72.33 & 71.27 & 65.24 & - & 81.22 & 80.79 & 79.55 & 72.81 & - \\
\hline 302-N120-FR & 6.00 & 151.82 & 149.57 & 142.70 & 127.08 & - & 178.87 & 178.22 & 172.44 & 153.72 & - \\
\hline 302-N150-FR & 1.99 & 21.50 & 21.45 & 21.40 & 20.29 & - & 21.06 & 20.92 & 20.68 & 19.31 & - \\
\hline 302-N150-FR & 4.00 & 77.49 & 77.30 & 75.84 & 68.82 & - & 88.97 & 88.51 & 86.37 & 77.28 & - \\
\hline 302-N150-FR & 6.00 & 159.25 & 157.03 & 148.66 & 134.63 & - & 196.06 & 194.07 & 185.02 & 162.437 & - \\
\hline
\end{tabular}


(b) $a / h$ for offset circular web opening case

\begin{tabular}{|c|c|c|c|c|c|c|c|c|c|}
\hline \multirow[t]{2}{*}{ Specimen } & \multirow{2}{*}{$\begin{array}{c}\text { Thickness } \\
t \\
(\mathrm{~mm})\end{array}$} & \multicolumn{4}{|c|}{ Unfastened FEA load per web, $\mathrm{P}_{\mathrm{FEA}}$} & \multicolumn{4}{|c|}{ Fastened FEA load per web, $\mathrm{P}_{\text {FEA }}$} \\
\hline & & $\begin{array}{l}A(0) \\
(k N)\end{array}$ & $\begin{array}{c}A(0.2) \\
(k N)\end{array}$ & $\begin{array}{c}A(0.4) \\
(k N)\end{array}$ & $\begin{array}{c}A(0.6) \\
(k N)\end{array}$ & $\begin{array}{l}A(0) \\
(k N)\end{array}$ & $\begin{array}{c}A(0.2) \\
(k N)\end{array}$ & $\begin{array}{c}A(0.4) \\
(k N)\end{array}$ & $\begin{array}{c}A(0.6) \\
(k N)\end{array}$ \\
\hline 142-N100-FR & 1.27 & 8.47 & 8.44 & 8.27 & 8.14 & 9.40 & 9.37 & 9.32 & 9.24 \\
\hline 142-N100-FR & 4.00 & 66.60 & 66.54 & 66.20 & 65.49 & 80.85 & 80.83 & 80.78 & 80.74 \\
\hline 142-N100-FR & 6.00 & 121.33 & 120.59 & 118.39 & 114.61 & 155.17 & 154.74 & 153.12 & 142.44 \\
\hline 142-N120-FR & 1.27 & 8.89 & 8.88 & 8.80 & 8.66 & 9.90 & 9.86 & 9.82 & 9.76 \\
\hline 142-N120-FR & 4.00 & 69.52 & 69.40 & 68.93 & 67.89 & 86.20 & 86.18 & 86.11 & 85.83 \\
\hline 142-N120-FR & 6.00 & 120.01 & 119.35 & 117.73 & 114.98 & 155.65 & 155.18 & 153.51 & 142.84 \\
\hline 142-N150-FR & 1.28 & 9.61 & 9.59 & 9.48 & 9.32 & 10.52 & 10.50 & 10.47 & 10.45 \\
\hline 142-N150-FR & 4.00 & 69.82 & 69.41 & 68.27 & 66.25 & 91.06 & 91.02 & 90.85 & 90.16 \\
\hline 142-N150-FR & 6.00 & 114.61 & 113.88 & 112.10 & 109.53 & 152.49 & 152.09 & 150.57 & 142.00 \\
\hline 202-N100-FR & 1.39 & 9.43 & 9.35 & 7.95 & 7.76 & 10.63 & 10.60 & 10.51 & 10.38 \\
\hline 202-N100-FR & 4.00 & 67.75 & 67.60 & 67.13 & 66.11 & 78.80 & 78.76 & 78.72 & 78.69 \\
\hline 202-N100-FR & 6.00 & 136.09 & 135.62 & 133.92 & 128.96 & 167.89 & 167.88 & 167.76 & 166.51 \\
\hline 202-N120-FR & 1.39 & 9.95 & 9.89 & 9.71 & 9.48 & 10.60 & 10.57 & 10.51 & 10.39 \\
\hline 202-N120-FR & 4.00 & 70.69 & 70.46 & 69.91 & 68.69 & 84.52 & 84.50 & 84.49 & 84.44 \\
\hline 202-N120-FR & 6.00 & 137.96 & 137.13 & 134.60 & 130.87 & 177.96 & 177.92 & 177.66 & 169.46 \\
\hline 202-N150-FR & 1.39 & 10.74 & 10.68 & 10.49 & 10.10 & 12.16 & 12.14 & 12.09 & 12.01 \\
\hline 202-N150-FR & 4.00 & 74.59 & 74.40 & 73.69 & 72.19 & 92.62 & 92.61 & 92.57 & 92.41 \\
\hline 202-N150-FR & 6.00 & 139.46 & 138.88 & 137.06 & 132.55 & 185.28 & 184.93 & 183.42 & 170.70 \\
\hline 302-N100-FR & 1.98 & 17.02 & 16.94 & 16.80 & 16.64 & 19.25 & 19.23 & 19.16 & 19.05 \\
\hline 302-N100-FR & 2.00 & 69.18 & 69.08 & 68.69 & 67.88 & 76.40 & 76.35 & 76.30 & 76.22 \\
\hline 302-N100-FR & 4.00 & 146.80 & 146.49 & 145.30 & 141.51 & 168.78 & 168.74 & 168.72 & 168.65 \\
\hline 302-N120-FR & 1.98 & 17.95 & 17.92 & 17.84 & 17.66 & 19.80 & 19.77 & 19.72 & 19.66 \\
\hline 302-N120-FR & 2.00 & 72.56 & 72.47 & 71.98 & 71.03 & 81.28 & 81.25 & 81.19 & 81.09 \\
\hline 302-N120-FR & 4.00 & 151.85 & 151.49 & 149.92 & 143.33 & 179.93 & 179.90 & 179.87 & 179.64 \\
\hline 302-N150-FR & 1.99 & 19.62 & 19.60 & 19.52 & 19.26 & 20.96 & 20.93 & 20.92 & 20.46 \\
\hline 302-N150-FR & 2.00 & 77.49 & 77.36 & 76.86 & 75.35 & 88.97 & 88.95 & 88.85 & 88.32 \\
\hline 302-N150-FR & 4.00 & 159.31 & 158.75 & 156.15 & 149.66 & 196.16 & 196.06 & 195.80 & 194.57 \\
\hline
\end{tabular}


(c) $x / h$ for offset circular web opening case

\begin{tabular}{|c|c|c|c|c|c|c|c|c|c|}
\hline \multirow[t]{2}{*}{ Specimen } & \multirow{2}{*}{$\begin{array}{c}\text { Thickness } \\
t \\
(\mathrm{~mm})\end{array}$} & \multicolumn{4}{|c|}{ Unfastened FEA load per web, $P($ FEA $)$} & \multicolumn{4}{|c|}{ Fastened FEA load per web, $\mathrm{P}_{\mathrm{FEA}}$} \\
\hline & & $\begin{array}{l}X(0) \\
(k N)\end{array}$ & $\begin{array}{c}X(0.2) \\
(k N)\end{array}$ & $\begin{array}{c}X(0.4) \\
(k N)\end{array}$ & $\begin{array}{c}X(0.6) \\
(k N)\end{array}$ & $\begin{array}{l}X(0) \\
(k N)\end{array}$ & $\begin{array}{c}X(0.2) \\
(k N)\end{array}$ & $\begin{array}{c}X(0.4) \\
(k N)\end{array}$ & $\begin{array}{c}X(0.6) \\
(k N)\end{array}$ \\
\hline 142-N100-A0-FR & 1.27 & 8.17 & 8.17 & 8.17 & 8.17 & 8.85 & 8.85 & 8.85 & 8.85 \\
\hline 142-N100-A0.2-FR & 1.27 & 8.01 & 8.02 & 8.03 & 8.07 & 8.81 & 8.82 & 8.83 & 8.83 \\
\hline 142-N100-A0.4-FR & 1.27 & 7.77 & 7.78 & 7.79 & 7.89 & 8.71 & 8.72 & 8.74 & 8.78 \\
\hline 142-N100-A0.6-FR & 1.27 & 7.26 & 7.32 & 7.53 & 7.73 & 8.24 & 8.34 & 8.54 & 8.69 \\
\hline 142-N100-A0.8-FR & 1.27 & 6.24 & 6.80 & 7.21 & 7.53 & -- & --- & --- & -- \\
\hline 142-N120-A0-FR & 1.27 & 8.65 & 8.65 & 8.65 & 8.65 & 9.42 & 9.42 & 9.42 & 9.42 \\
\hline 142-N120-A0.2-FR & 1.27 & 8.50 & 8.56 & 8.57 & 8.60 & 9.38 & 9.39 & 9.40 & 9.41 \\
\hline 142-N120-A0.4-FR & 1.27 & 8.20 & 8.25 & 8.34 & 8.45 & 9.26 & 9.28 & 9.30 & 9.36 \\
\hline 142-N120-A0.6-FR & 1.27 & 7.66 & 7.79 & 8.04 & 8.26 & 8.78 & 8.94 & 9.16 & 9.28 \\
\hline 142-N120-A0.8-FR & 1.27 & 6.57 & 7.14 & 7.63 & 7.98 & 8.05 & 8.41 & 8.96 & 9.19 \\
\hline 142-N150-A0-FR & 1.28 & 9.43 & 9.43 & 9.43 & 9.43 & 10.17 & 10.17 & 10.17 & 10.17 \\
\hline 142-N150-A0.2-FR & 1.28 & 9.28 & 9.31 & 9.33 & 9.36 & 10.12 & 10.13 & 10.14 & 10.16 \\
\hline 142-N150-A0.4-FR & 1.28 & 8.93 & 8.94 & 9.06 & 9.19 & 9.95 & 9.97 & 10.06 & 10.12 \\
\hline 142-N150-A0.6-FR & 1.28 & 8.13 & 8.38 & 8.68 & 8.94 & 9.37 & 9.64 & 9.91 & 10.06 \\
\hline 142-N150-A0.8-FR & 1.28 & 7.05 & 7.61 & 8.09 & 8.45 & 8.17 & 8.97 & 9.55 & 9.89 \\
\hline 202-N100-A0-FR & 1.39 & 9.33 & 9.33 & 9.33 & 9.33 & 9.61 & 9.61 & 9.61 & 9.61 \\
\hline 202-N100-A0.2-FR & 1.39 & 9.18 & 9.20 & 9.21 & 9.23 & 8.53 & 9.55 & 9.57 & 9.60 \\
\hline 202-N100-A0.4-FR & 1.39 & 8.83 & 8.84 & 8.94 & 9.06 & 8.93 & 8.94 & 9.07 & 9.25 \\
\hline 202-N100-A0.6-FR & 1.39 & 8.13 & 8.29 & 8.54 & 8.78 & 8.31 & 8.40 & 8.84 & 9.05 \\
\hline 202-N120-A0-FR & 1.39 & 9.91 & 9.91 & 9.91 & 9.91 & 10.82 & 10.82 & 10.82 & 10.82 \\
\hline 202-N120-A0.2-FR & 1.39 & 9.76 & 9.79 & 9.81 & 9.83 & 10.74 & 10.77 & 10.79 & 10.81 \\
\hline 202-N120-A0.4-FR & 1.39 & 9.36 & 9.37 & 9.49 & 9.62 & 10.52 & 10.52 & 10.62 & 10.74 \\
\hline 202-N120-A0.6-FR & 1.39 & 8.42 & 8.66 & 8.99 & 9.30 & 9.92 & 10.15 & 10.49 & 10.64 \\
\hline 202-N150-A0-FR & 1.45 & 11.63 & 11.63 & 11.63 & 11.63 & 11.90 & 11.90 & 11.90 & 11.90 \\
\hline 202-N150-A0.2-FR & 1.45 & 11.47 & 11.49 & 11.51 & 11.55 & 11.85 & 11.86 & 11.88 & 11.89 \\
\hline 202-N150-A0.4-FR & 1.45 & 10.86 & 10.92 & 11.09 & 11.26 & 11.52 & 11.60 & 11.78 & 11.84 \\
\hline 202-N150-A0.6-FR & 1.45 & 9.67 & 9.98 & 10.37 & 10.76 & 10.73 & 11.09 & 11.56 & 11.71 \\
\hline 302-N100-A0-FR & 1.98 & 17.94 & 17.94 & 17.94 & 17.94 & 18.62 & 18.62 & 18.62 & 18.62 \\
\hline 302-N100-A0.2-FR & 1.98 & 17.73 & 17.76 & 17.79 & 17.84 & 18.54 & 18.57 & 18.59 & 18.61 \\
\hline 302-N120-A0-FR & 1.96 & 18.67 & 18.67 & 18.67 & 18.67 & 19.27 & 19.27 & 19.27 & 19.27 \\
\hline 302-N120-A0.2-FR & 1.96 & 18.50 & 18.54 & 18.59 & 18.63 & 19.23 & 19.24 & 19.52 & 19.63 \\
\hline 302-N120-A0.4-FR & 1.96 & 18.04 & 18.06 & 18.30 & 18.49 & 18.42 & 18.55 & 18.70 & 18.71 \\
\hline 302-N120-A0.6-FR & 1.96 & 16.87 & 17.75 & 18.72 & 19.43 & 18.08 & 18.15 & 18.22 & 18.39 \\
\hline 302-N150-A0-FR & 1.99 & 21.14 & 21.14 & 21.14 & 21.14 & 20.57 & 20.57 & 20.57 & 20.57 \\
\hline $302-\mathrm{N} 150-\mathrm{A} 0.2-\mathrm{FR}$ & 1.99 & 19.97 & 21.00 & 21.05 & 21.10 & 20.49 & 20.51 & 20.52 & 20.54 \\
\hline 302-N150-A0.4-FR & 1.99 & 20.34 & 20.37 & 20.68 & 20.92 & 20.27 & 20.39 & 20.40 & 20.45 \\
\hline 302-N150-A0.6-FR & 1.99 & 18.56 & 19.15 & 19.99 & 20.58 & 19.03 & 19.75 & 19.88 & 20.16 \\
\hline
\end{tabular}




\section{Appendix B: Ferritic}

Table B1 Web crippling strengths of ferritic stainless steel sections predicted from finite element analysis (a) $a / h$ for centred circular web opening case

\begin{tabular}{|c|c|c|c|c|c|c|c|c|c|c|c|}
\hline \multirow[t]{2}{*}{ Specimen } & \multirow{2}{*}{$\begin{array}{c}\text { Thickness } \\
t \\
(\mathrm{~mm})\end{array}$} & \multicolumn{5}{|c|}{ Unfastened FEA load per web, $\mathrm{P}_{\mathrm{FEA}}$} & \multicolumn{5}{|c|}{ Fastened FEA load per web, $\mathrm{P}_{\mathrm{FEA}}$} \\
\hline & & $\begin{array}{l}A(0) \\
(k N)\end{array}$ & $\begin{array}{c}A(0.2) \\
(k N)\end{array}$ & $\begin{array}{c}A(0.4) \\
(k N)\end{array}$ & $\begin{array}{c}A(0.6) \\
(k N)\end{array}$ & $\begin{array}{c}A(0.8) \\
(\mathrm{kN})\end{array}$ & $\begin{array}{l}A(0) \\
(k N)\end{array}$ & $\begin{array}{c}A(0.2) \\
(k N)\end{array}$ & $\begin{array}{c}A(0.4) \\
(k N)\end{array}$ & $\begin{array}{c}A(0.6) \\
(k N)\end{array}$ & $\begin{array}{c}A(0.8) \\
(k N)\end{array}$ \\
\hline 142-N100-FR & 1.27 & 9.64 & 9.55 & 9.50 & 8.98 & 7.98 & 10.13 & 10.01 & 9.83 & 9.57 & 8.56 \\
\hline 142-N100-FR & 4.00 & 75.39 & 74.54 & 70.34 & 64.89 & 56.30 & 86.65 & 86.32 & 83.06 & 78.31 & 70.35 \\
\hline 142-N100-FR & 6.00 & 137.93 & 136.61 & 124.50 & 109.57 & 88.79 & 170.00 & 167.14 & 158.77 & 145.91 & 135.41 \\
\hline 142-N120-FR & 1.27 & 10.30 & 10.25 & 10.23 & 9.38 & 8.37 & 11.01 & 10.99 & 10.88 & 10.17 & 9.03 \\
\hline 142-N120-FR & 4.00 & 78.92 & 77.99 & 73.93 & 67.87 & 56.96 & 94.74 & 94.16 & 89.69 & 82.18 & 73.45 \\
\hline 142-N120-FR & 6.00 & 138.00 & 134.32 & 124.67 & 108.57 & 87.95 & 172.45 & 168.87 & 160.43 & 148.22 & 130.58 \\
\hline 142-N150-FR & 1.28 & 11.15 & 11.12 & 10.69 & 9.77 & 8.80 & 11.91 & 11.94 & 11.64 & 10.72 & 9.62 \\
\hline 142-N150-FR & 4.00 & 79.09 & 78.31 & 74.62 & 66.02 & 53.18 & 102.89 & 101.57 & 101.57 & 90.09 & 80.21 \\
\hline 142-N150-FR & 6.00 & 132.05 & 129.07 & 120.00 & 103.68 & 82.86 & 170.58 & 167.70 & 160.53 & 149.33 & 133.15 \\
\hline 202-N100-FR & 1.39 & 10.95 & 10.93 & 10.92 & 9.86 & - & 10.99 & 10.97 & 10.56 & 10.47 & - \\
\hline 202-N100-FR & 4.00 & 76.67 & 76.06 & 73.11 & 66.17 & - & 84.44 & 84.09 & 82.29 & 75.25 & - \\
\hline 202-N100-FR & 6.00 & 153.20 & 150.53 & 142.09 & 128.00 & - & 179.07 & 177.81 & 172.61 & 155.54 & - \\
\hline 202-N120-FR & 1.39 & 11.59 & 11.58 & 11.25 & 10.05 & - & 12.51 & 12.37 & 12.24 & 11.90 & - \\
\hline 202-N120-FR & 4.00 & 80.57 & 79.82 & 75.72 & 69.10 & - & 92.59 & 92.32 & 88.81 & 79.69 & - \\
\hline 202-N120-FR & 6.00 & 158.38 & 157.84 & 146.08 & 129.79 & - & 194.15 & 192.76 & 184.90 & 166.88 & - \\
\hline 202-N150-FR & 1.39 & 12.25 & 12.18 & 11.52 & 10.38 & - & 13.73 & 13.46 & 13.14 & 12.49 & - \\
\hline 202-N150-FR & 4.00 & 85.51 & 84.46 & 79.56 & 73.90 & - & 104.08 & 102.52 & 96.97 & 88.13 & - \\
\hline 202-N150-FR & 6.00 & 161.20 & 158.91 & 149.02 & 128.86 & - & 213.37 & 204.50 & 193.90 & 175.41 & - \\
\hline 302-N100-FR & 1.98 & 20.65 & 20.60 & 20.55 & 20.41 & - & 20.91 & 20.89 & 20.26 & 19.91 & - \\
\hline 302-N100-FR & 4.00 & 77.78 & 77.71 & 76.25 & 72.26 & - & 80.76 & 80.64 & 78.58 & 75.58 & - \\
\hline 302-N100-FR & 6.00 & 163.56 & 162.23 & 159.46 & 141.16 & - & 175.68 & 175.16 & 169.02 & 159.23 & - \\
\hline 302-N120-FR & 1.98 & 22.47 & 22.24 & 22.16 & 21.04 & - & 21.96 & 21.95 & 21.80 & 20.62 & - \\
\hline 302-N120-FR & 4.00 & 82.48 & 82.46 & 81.75 & 74.75 & - & 87.93 & 87.73 & 86.99 & 79.95 & - \\
\hline 302-N120-FR & 6.00 & 171.22 & 169.60 & 163.26 & 146.01 & - & 192.12 & 191.29 & 187.23 & 166.10 & - \\
\hline 302-N150-FR & 1.99 & 24.65 & 24.55 & 24.47 & 22.01 & - & 23.59 & 23.57 & 23.26 & 21.28 & - \\
\hline 302-N150-FR & 4.00 & 88.92 & 88.82 & 86.90 & 78.24 & - & 97.97 & 97.61 & 95.84 & 84.30 & - \\
\hline 302-N150-FR & 6.00 & 181.23 & 179.42 & 170.46 & 154.64 & - & 215.29 & 213.67 & 205.54 & 174.956 & - \\
\hline
\end{tabular}


(b) $a / h$ for offset circular web opening case

\begin{tabular}{|c|c|c|c|c|c|c|c|c|c|}
\hline \multirow[t]{3}{*}{ Specimen } & \multirow{3}{*}{$\begin{array}{c}\text { Thickness } \\
t \\
(\mathrm{~mm})\end{array}$} & \multicolumn{4}{|c|}{ Unfastened FEA load per web, $P_{\mathrm{FEA}}$} & \multicolumn{4}{|c|}{ Fastened FEA load per web, $\mathrm{P}_{\mathrm{FEA}}$} \\
\hline & & $A(0)$ & $A(0.2)$ & $A(0.4)$ & $A(0.6)$ & $A(0)$ & $A(0.2)$ & $A(0.4)$ & $A(0.6)$ \\
\hline & & $(k N)$ & $(k N)$ & $(k N)$ & $(\mathrm{kN})$ & $(k N)$ & $(k N)$ & $(k N)$ & $(k N)$ \\
\hline 142-N100-FR & 1.27 & 9.54 & 9.53 & 9.48 & 9.40 & 10.18 & 10.17 & 10.16 & 10.15 \\
\hline 142-N100-FR & 4.00 & 75.44 & 75.36 & 75.06 & 74.56 & 86.75 & 86.73 & 86.71 & 86.69 \\
\hline 142-N100-FR & 6.00 & 135.73 & 135.39 & 134.40 & 132.63 & 170.00 & 169.78 & 168.93 & 159.64 \\
\hline 142-N120-FR & 1.27 & 10.28 & 10.27 & 10.18 & 10.02 & 11.01 & 10.99 & 10.98 & 10.96 \\
\hline 142-N120-FR & 4.00 & 78.98 & 78.87 & 78.50 & 77.69 & 94.82 & 94.78 & 94.76 & 94.74 \\
\hline 142-N120-FR & 6.00 & 137.05 & 136.63 & 135.45 & 133.41 & 172.45 & 172.20 & 171.01 & 160.33 \\
\hline 142-N150-FR & 1.28 & 11.14 & 11.10 & 10.94 & 10.61 & 11.95 & 11.93 & 11.91 & 11.88 \\
\hline 142-N150-FR & 4.00 & 79.05 & 78.84 & 78.31 & 77.34 & 102.50 & 102.49 & 102.38 & 102.05 \\
\hline 142-N150-FR & 6.00 & 131.75 & 131.24 & 129.91 & 127.68 & 170.58 & 170.31 & 169.05 & 160.25 \\
\hline 202-N100-FR & 1.39 & 10.74 & 10.67 & 10.44 & 9.83 & 11.61 & 11.59 & 11.57 & 11.50 \\
\hline 202-N100-FR & 4.00 & 76.62 & 76.48 & 76.03 & 75.16 & 84.49 & 84.47 & 84.44 & 84.41 \\
\hline 202-N100-FR & 6.00 & 153.08 & 152.73 & 151.58 & 148.79 & 179.11 & 179.08 & 179.05 & 179.01 \\
\hline 202-N120-FR & 1.39 & 11.40 & 11.29 & 10.88 & 10.03 & 11.89 & 11.86 & 11.82 & 11.70 \\
\hline 202-N120-FR & 4.00 & 80.53 & 80.37 & 79.81 & 78.62 & 92.59 & 92.58 & 92.54 & 92.50 \\
\hline 202-N120-FR & 6.00 & 157.32 & 156.91 & 155.42 & 151.66 & 194.15 & 194.11 & 194.04 & 190.53 \\
\hline 202-N150-FR & 1.39 & 12.08 & 11.88 & 11.27 & 10.49 & 13.75 & 13.74 & 13.69 & 13.52 \\
\hline 202-N150-FR & 4.00 & 85.49 & 85.29 & 84.57 & 82.58 & 104.47 & 103.42 & 103.39 & 103.26 \\
\hline 202-N150-FR & 6.00 & 161.15 & 160.58 & 158.72 & 154.05 & 207.25 & 206.87 & 205.31 & 192.39 \\
\hline 302-N100-FR & 1.98 & 19.02 & 19.00 & 18.94 & 18.81 & 20.93 & 20.91 & 20.87 & 20.78 \\
\hline 302-N100-FR & 2.00 & 77.78 & 77.69 & 77.34 & 76.55 & 80.83 & 80.80 & 80.75 & 80.69 \\
\hline 302-N100-FR & 4.00 & 163.58 & 163.28 & 162.22 & 158.58 & 175.77 & 175.73 & 175.71 & 175.67 \\
\hline 302-N120-FR & 1.98 & 20.63 & 20.52 & 20.43 & 20.18 & 21.96 & 21.95 & 21.93 & 21.84 \\
\hline 302-N120-FR & 2.00 & 82.48 & 82.37 & 81.86 & 80.59 & 87.97 & 87.95 & 87.90 & 87.81 \\
\hline 302-N120-FR & 4.00 & 171.25 & 170.87 & 169.26 & 160.22 & 192.17 & 192.16 & 192.13 & 192.06 \\
\hline 302-N150-FR & 1.99 & 22.47 & 22.45 & 22.25 & 21.50 & 23.59 & 23.59 & 23.56 & 23.41 \\
\hline 302-N150-FR & 2.00 & 88.79 & 88.60 & 87.89 & 85.33 & 97.97 & 97.96 & 97.94 & 97.75 \\
\hline 302-N150-FR & 4.00 & 181.04 & 180.29 & 176.64 & 166.49 & 215.31 & 215.30 & 215.29 & 215.25 \\
\hline
\end{tabular}


(c) $x / h$ for offset circular web opening case

\begin{tabular}{|c|c|c|c|c|c|c|c|c|c|}
\hline \multirow[t]{2}{*}{ Specimen } & \multirow{2}{*}{$\begin{array}{c}\text { Thickness } \\
t \\
(\mathrm{~mm})\end{array}$} & \multicolumn{4}{|c|}{ Unfastened FEA load per web, $P($ FEA $)$} & \multicolumn{4}{|c|}{ Fastened FEA load per web, $\mathrm{P}_{\mathrm{FEA}}$} \\
\hline & & $\begin{array}{l}X(0) \\
(k N)\end{array}$ & $\begin{array}{c}X(0.2) \\
(k N)\end{array}$ & $\begin{array}{c}X(0.4) \\
(k N)\end{array}$ & $\begin{array}{c}X(0.6) \\
(k N)\end{array}$ & $\begin{array}{l}X(0) \\
(k N)\end{array}$ & $\begin{array}{c}X(0.2) \\
(k N)\end{array}$ & $\begin{array}{c}X(0.4) \\
(k N)\end{array}$ & $\begin{array}{c}X(0.6) \\
(k N)\end{array}$ \\
\hline 142-N100-A0-FR & 1.27 & 9.19 & 9.19 & 9.19 & 9.19 & 9.67 & 9.67 & 9.67 & 9.67 \\
\hline 142-N100-A0.2-FR & 1.27 & 9.10 & 9.11 & 9.12 & 9.15 & 9.62 & 9.64 & 9.65 & 9.66 \\
\hline 142-N100-A0.4-FR & 1.27 & 8.83 & 8.85 & 8.95 & 9.05 & 9.58 & 9.59 & 9.61 & 9.65 \\
\hline 142-N100-A0.6-FR & 1.27 & 8.31 & 8.45 & 8.69 & 8.91 & 9.21 & 9.34 & 9.53 & 9.63 \\
\hline 142-N100-A0.8-FR & 1.27 & 7.14 & 7.76 & 8.23 & 8.62 & --- & --- & --- & -- \\
\hline 142-N120-A0-FR & 1.27 & 9.96 & 9.96 & 9.96 & 9.96 & 10.56 & 10.56 & 10.56 & 10.56 \\
\hline 142-N120-A0.2-FR & 1.27 & 9.84 & 9.86 & 9.87 & 9.90 & 10.51 & 10.52 & 10.52 & 10.54 \\
\hline 142-N120-A0.4-FR & 1.27 & 9.48 & 9.51 & 9.63 & 9.75 & 10.36 & 10.38 & 10.43 & 10.50 \\
\hline 142-N120-A0.6-FR & 1.27 & 8.71 & 8.92 & 9.22 & 9.50 & 9.83 & 10.04 & 10.30 & 10.45 \\
\hline 142-N120-A0.8-FR & 1.27 & 7.46 & 8.09 & 8.56 & 8.90 & 8.32 & 9.31 & 10.04 & 10.39 \\
\hline 142-N150-A0-FR & 1.28 & 11.29 & 11.29 & 11.29 & 11.29 & 11.57 & 11.57 & 11.57 & 11.57 \\
\hline 142-N150-A0.2-FR & 1.28 & 10.72 & 10.75 & 10.77 & 10.81 & 11.49 & 11.50 & 11.53 & 11.55 \\
\hline 142-N150-A0.4-FR & 1.28 & 10.18 & 10.21 & 10.78 & 10.94 & 11.23 & 11.25 & 11.39 & 11.50 \\
\hline 142-N150-A0.6-FR & 1.28 & 9.19 & 9.48 & 9.82 & 10.13 & 10.49 & 10.78 & 11.16 & 11.40 \\
\hline 142-N150-A0.8-FR & 1.28 & 7.94 & 8.53 & 8.99 & 9.25 & 8.99 & 9.87 & 10.62 & 11.18 \\
\hline 202-N100-A0-FR & 1.39 & 10.61 & 10.61 & 10.61 & 10.61 & 10.58 & 10.58 & 10.58 & 10.58 \\
\hline 202-N100-A0.2-FR & 1.39 & 10.46 & 10.50 & 10.51 & 10.54 & 10.53 & 10.55 & 10.57 & 10.58 \\
\hline 202-N100-A0.4-FR & 1.39 & 10.01 & 10.03 & 10.16 & 10.31 & 9.90 & 9.92 & 10.05 & 10.14 \\
\hline 202-N100-A0.6-FR & 1.39 & 8.84 & 9.13 & 9.44 & 9.74 & 9.55 & 9.72 & 9.88 & 10.06 \\
\hline 202-N120-A0-FR & 1.39 & 11.36 & 11.36 & 11.36 & 11.36 & 12.10 & 12.10 & 12.10 & 12.10 \\
\hline 202-N120-A0.2-FR & 1.39 & 11.17 & 11.20 & 11.20 & 11.23 & 12.05 & 12.06 & 12.07 & 12.05 \\
\hline 202-N120-A0.4-FR & 1.39 & 10.38 & 10.46 & 10.63 & 10.80 & 11.76 & 11.77 & 11.94 & 12.05 \\
\hline 202-N120-A0.6-FR & 1.39 & 9.06 & 9.35 & 9.65 & 9.93 & 11.04 & 11.28 & 11.70 & 11.98 \\
\hline 202-N150-A0-FR & 1.45 & 13.16 & 13.16 & 13.16 & 13.16 & 13.45 & 13.45 & 13.45 & 13.45 \\
\hline 202-N150-A0.2-FR & 1.45 & 12.85 & 12.88 & 12.89 & 12.93 & 13.34 & 13.35 & 13.40 & 13.44 \\
\hline 202-N150-A0.4-FR & 1.45 & 11.76 & 11.85 & 12.03 & 12.24 & 12.86 & 12.91 & 13.16 & 13.35 \\
\hline 202-N150-A0.6-FR & 1.45 & 10.29 & 10.58 & 10.89 & 11.22 & 11.86 & 12.21 & 12.75 & 13.21 \\
\hline 302-N100-A0-FR & 1.98 & 20.09 & 20.09 & 20.09 & 20.09 & 20.12 & 20.12 & 20.12 & 20.12 \\
\hline 302-N100-A0.2-FR & 1.98 & 19.94 & 19.99 & 20.03 & 20.07 & 20.05 & 20.09 & 20.11 & 20.12 \\
\hline 302-N120-A0-FR & 1.96 & 21.37 & 21.37 & 21.37 & 21.37 & 21.36 & 21.36 & 21.36 & 21.36 \\
\hline 302-N120-A0.2-FR & 1.96 & 21.20 & 21.22 & 21.27 & 21.32 & 21.34 & 21.34 & 21.33 & 21.34 \\
\hline 302-N120-A0.4-FR & 1.96 & 20.51 & 20.53 & 20.82 & 21.10 & 20.36 & 20.56 & 20.68 & 20.67 \\
\hline 302-N120-A0.6-FR & 1.96 & 18.25 & 18.88 & 19.79 & 20.63 & 19.95 & 20.07 & 20.17 & 20.34 \\
\hline 302-N150-A0-FR & 1.99 & 24.20 & 24.20 & 24.20 & 24.20 & 23.17 & 23.17 & 23.17 & 23.17 \\
\hline $302-\mathrm{N} 150-\mathrm{A} 0.2-\mathrm{FR}$ & 1.99 & 23.91 & 23.94 & 24.01 & 24.10 & 23.12 & 23.13 & 23.15 & 23.19 \\
\hline 302-N150-A0.4-FR & 1.99 & 22.58 & 22.71 & 23.12 & 23.60 & 22.42 & 22.80 & 22.97 & 22.97 \\
\hline 302-N150-A0.6-FR & 1.99 & 19.85 & 20.53 & 21.54 & 22.57 & 20.73 & 21.73 & 22.16 & 22.37 \\
\hline
\end{tabular}

